\title{
Synthesis, characterization and in vitro biological evaluation of some new 1,3,5-triazine-chalcone hybrid molecules as Mycobacterium tuberculosis H37Rv inhibitors
}

\author{
Subbarayal Reddy Dwarampudi a,*, Gowri Sankar Dannana a, \\ Vasudeva Rao Avupati b and Venkata Satyanarayana Murthy Bendi c
}

a Pharmaceutical Analysis and Quality Assurance Division, Andhra University College of Pharmaceutical Sciences, Andhra University, Visakhapatnam-530003, Andhra Pradesh, India

b Pharmaceutical Chemistry Division, Andhra University College of Pharmaceutical Sciences, Andhra University, Visakhapatnam-530003, Andhra Pradesh, India c Pharmacology Division, Andhra University College of Pharmaceutical Sciences, Andhra University, Visakhapatnam-530003, Andhra Pradesh, India

*Corresponding author at: Pharmaceutical Analysis and Quality Assurance Division, Andhra University College of Pharmaceutical Sciences, Andhra University, Visakhapatnam-530003, Andhra Pradesh, India.

Tel.: +91.94.40653159. Fax: +91.891.2755075. E-mail address: dwarampudisubbarayalreddy@gmail.com (D.S. Reddy).

\section{ARTICLE INFORMATION}

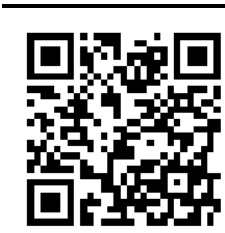

DOI: $10.5155 /$ eurjchem.5.4.570-576.1098

Received: 05 June 2014

Received in revised form: 30 June 2014

Accepted: 02 July 2014

Online: 31 December 2014

\section{KEYWORDS}

Hybrid molecules

Alamar blue assay

1,3,5-Triaizine-chalcone

Structure activity relationship

Minimum inhibitory concentration

Mycobacterium tuberculosis H37Rv

\section{Introduction}

Tuberculosis (TB) remains a major global health problem. In 2012, an estimated 8.6 million people developed TB and 1.3 million died from the disease (including 320000 deaths among HIV-positive people) [1]. It is caused by several species of Mycobacteria including Mycobacterium tuberculosis, M. bovis, $M$. africanum, M. microti, M. leprae and M. avium that are intracellular, Gram-positive, non-motile, and rod-shaped obligate aerobic pathogens of higher vertebrates [2]. Although this disease can be cured with the current therapy, the treatments require six to nine months of time period that is too long, and accompanied by significant toxicity [3]. These factors make patient compliance to therapy very difficult, and this noncompliance frequently selects for drug-resistant TB bacteria [4]. An increasing occurrence of deaths due to tuberculosis and the known drawbacks of the current existing drugs including the emergence of multi drug-resistant strains have led to a renewed interest in the discovery of new antitubercular agents with novel modes of actions [5]. The topical researches focused on new synthetic products that have shown a useful way to obtain a potentially rich source of drug candidates [6-9].

In recent past, 2,4,6-trisubstituted-1,3,5-triazine scaffolds were discovered as a potent inhibitors of Mycobacterium tuberculosis (Mtb) H37Rv [10]. Currently 1,3,5-triazine derivatives have been found to possess wide range of biological activities, such as adenosine receptor antagonist [11], antiamoebic [12], anticancer [13], antileishmanial [14], antimalarial [15], antimicrobial [16], antiviral [17], antitubercular [18], carbonic anhydrase inhibitor [19], cathepsin B inhibitor [20], cholesteryl ester transfer protein inhibitor [21], corticotropin-releasing factor ligand [22], $\mathrm{CRF}_{1} \mathrm{PET}$ imaging agent [23] cytosolic phospholipase A2a inhibitor [24], dipeptidyl peptidase IV inhibitor [25], bacterial enzyme DNA helicase inhibitor [26], dual PI3/mTOR inhibitor [27], glucocerebrosidase inhibitor [28], $\alpha$-glucosidase inhibitor [29], growth factor inhibitor [30], human gonadotropin-releasing hormone receptor antagonist [31], 5- $\mathrm{HT}_{7}$ receptor antagonist [32], inosine monophosphate dehydrogenase inhibitor [33], mTOR kinase inhibitor [34], 


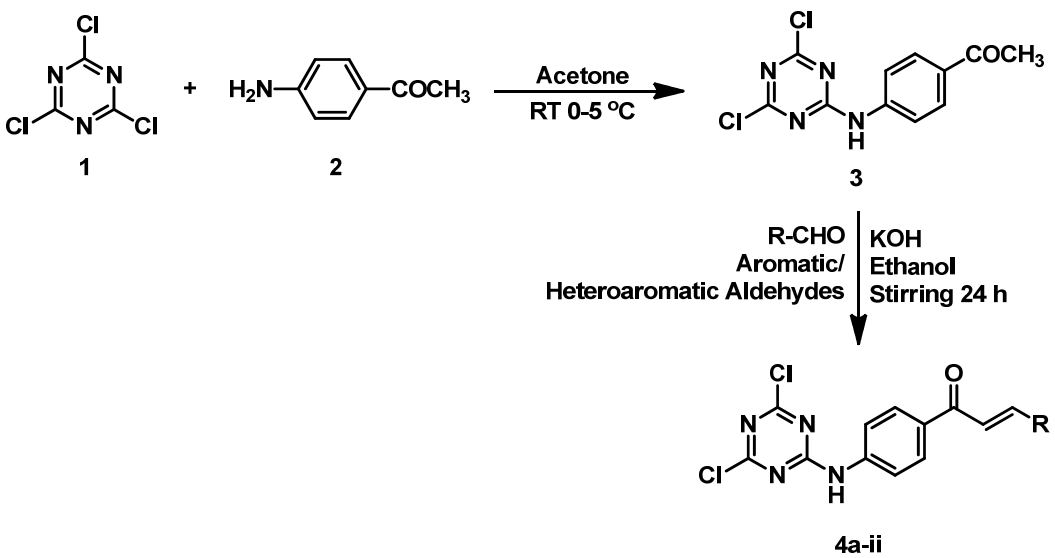

Scheme 1

voltage-gated sodium channel $\mathrm{Na}_{\mathrm{v}} 1.7$ antagonist [35], neuronal voltage-gated sodium channel blocker [36], phosphodiesterase type 4 inhibitor [37], protein kinase CK2 inhibitor [38], ROCK inhibitor [39], $\beta$-secretase inhibitor [40], sorbitol dehydrogenase inhibitor [41], tryptophan hydroxylase inhibitor [42] and VLA-4 integrin antagonist [43]. Similarly, azomethine moiety has gained a great importance, since it has been found to possess several biological activities, such as antimicrobial [4447], antiviral [48,49], antioxidant [50], radical inhibitor [51], antitumor [52,53], carbonic anhydrase inhibitor [54], xanthine oxidase inhibitor [55], antibacterial [56-59], plant growth regulator [60], free radical scavenger [61], trypsin inhibitor [62], inhibitor of cartilage matrix degeneration [63], 5- $\mathrm{HT}_{6}$ antagonist [64], anti-inflammatory [65] and analgesic [66,67]. Similarly, chalcones ( $\alpha, \beta$-unsatured ketones) captivated significant attention in drug discovery chemistry. Chalcones $(\alpha, \beta$-unsatured ketones) have gained huge significance as these compounds exhibit several biological activities, such as antimicrobial [68], antiviral [69], antioxidant [70], radical inhibitor [71], antitumor [72], carbonic anhydrase inhibitor [73], xanthine oxidase inhibitor [74], antibacterial [75], plant growth regulator [76], free radical scavenger [77], anti-inflammatory [78] and analgesic [79].

As a part of our on-going research in systematic investigation of synthesizing some novel bioactive compounds in relation to their Mtb H37Rv inhibitory activity, we prepared a series of some novel 1,3,5-triazine-chalcone hybrid molecules (4a-ii) [80]. However, we have found that 1,3,5-triazinechalcone hybrid molecules (4a-ii) have the considerable potential to act as a new class of Mtb H37Rv inhibitors, which can be obtained with the efficient methods in organic synthesis (Scheme 1). The novelty of this work is that none of the 1,3,5triazine-chalcone hybrid molecules (4a-ii) synthesized in the present study were earlier not reported to possess any inhibitory activity against Mtb H37Rv strain.

\section{Experimental}

\subsection{Instrumentation}

Melting points were taken in open capillary tubes and are therefore uncorrected. Purity of the compounds was checked on silica gel G TLC plates of $2 \mathrm{~mm}$ thickness using $n$-hexane and ethyl acetate as solvent system. The visualization of spot was carried out in an iodine chamber. The FT-IR spectra were recorded on Perkin-Elmer spectrometer. The ${ }^{1} \mathrm{H}$ NMR spectra were scanned on a Bruker $400 \mathrm{MHz}$. spectrometer in DMSO- $d_{6}$ using TMS as internal standard and chemical shifts are expressed in $\delta \mathrm{ppm}$. The electronspray ionisation mass spectra
(ESI-MS) were recorded on an Agilent 6100 QQQ mass spectrometer (positive ion mode). The UV-Vis absorption spectra of the compounds were recorded on a Hitachi U-1600 spectrophotometer.

\subsection{General procedure for the synthesis of 1,3,5-triazine- chalcone hybrid molecules (4a-ii)}

The reaction sequence intended for the preparation of title compounds (4a-ii) is shown in Scheme 1, and their physical properties are depicted in Table 1 . The chief intermediate in the present study 1-(4-(4,6-dichloro-1,3,5-triazin-2-ylamino) phenyl)ethanone (3) was prepared by reaction between cyanuric chloride i.e. 2,4,6-trichloro-1,3,5-triazine (1) and 4-aminoacetophenone (2) [10]. Further, successive base catalyzed Claisen-Schmidt condensation of the compound 3 with appropriate substituted aromatic/heteroaromatic aldehydes in the presence of $100 \%$ potassium hydroxide solution in ethanol afforded a series of 1-(4-(4,6-dichloro-1,3, 5-triazin-2-ylamino)phenyl)-3-(substituted)-2-propen-1-ones (4a-ii) in good yield. All the newly synthesized compounds were characterized by CHN elemental analysis and spectroscopic methods such as FT-IR, ${ }^{1} \mathrm{H}$ NMR, and LC mass spectral analysis. Eventually all the spectra of the new products (4a-ii) are in keeping with the predictable structures.

(E)-1-(4-(4,6-Dichloro-1,3,5-triazin-2-ylamino)phenyl)-3(phenyl)-2-propen-1-one (4a): Colour: Light yellow crystals. FTIR (KBr, $\left.v_{\max }, \mathrm{cm}^{-1}\right): 3155(\mathrm{~N}-\mathrm{H}), 3031(\mathrm{C}-\mathrm{H}$, aromatic), 2884 (C-H, aliphatic), $1688(\mathrm{C}=0), 1645(\mathrm{C}=\mathrm{C}$, aliphatic), $1513(\mathrm{C}=\mathrm{C}$, aromatic), $689(\mathrm{C}-\mathrm{Cl}) .{ }^{1} \mathrm{H}$ NMR $\left(400 \mathrm{MHz}, \mathrm{DMSO}-d_{6}, \delta, \mathrm{ppm}\right)$ : 7.13-7.74 (m, 9H, Ar-H), $7.78(\mathrm{~d}, J=15.2 \mathrm{~Hz}, 1 \mathrm{H}, \mathrm{HC}=\mathrm{CH}(\mathrm{H}-\alpha)$ ), $8.01(\mathrm{~d}, J=15.2 \mathrm{~Hz}, 1 \mathrm{H}, \mathrm{HC}=\mathrm{CH}(\mathrm{H}-\beta)), 9.74(\mathrm{~s}, 1 \mathrm{H}, \mathrm{NH})$. ESI-MS $(\mathrm{m} / \mathrm{z}): 372[\mathrm{M}+\mathrm{H}]^{+}$.

(E)-1-(4-(4,6-Dichloro-1,3,5-triazin-2-ylamino)phenyl)-3-(2methylphenyl)-2-propen-1-one (4b): Colour: Light yellow crystals. FT-IR $\left(\mathrm{KBr}, v_{\max }, \mathrm{cm}^{-1}\right): 3152(\mathrm{~N}-\mathrm{H}), 3022(\mathrm{C}-\mathrm{H}$ aromatic), 2881 (C-H, aliphatic), 1689 (C=0), 1623 (C=C, aliphatic), $1501\left(\mathrm{C}=\mathrm{C}\right.$, aromatic), $688(\mathrm{C}-\mathrm{Cl}) .{ }^{1} \mathrm{H}$ NMR $(400 \mathrm{MHz}$ DMSO- $\left.d_{6}, \delta, \mathrm{ppm}\right): 2.32\left(\mathrm{~s}, 3 \mathrm{H}, \mathrm{CH}_{3}\right), 7.43-8.04(\mathrm{~m}, 8 \mathrm{H}, \mathrm{Ar}-\mathrm{H})$, $7.78(\mathrm{~d}, J=15.2 \mathrm{~Hz}, 1 \mathrm{H}, \mathrm{HC}=\mathrm{CH}(\mathrm{H}-\alpha)), 8.01(\mathrm{~d}, J=15.2 \mathrm{~Hz}, 1 \mathrm{H}$, $\mathrm{HC}=\mathrm{CH}(\mathrm{H}-\beta)), 9.74(\mathrm{~s}, 1 \mathrm{H}, \mathrm{NH})$. ESI-MS $(\mathrm{m} / \mathrm{z}): 386[\mathrm{M}+\mathrm{H}]^{+}$.

(E)-1-(4-(4,6-Dichloro-1,3,5-triazin-2-ylamino)phenyl)-3(3-methylphenyl)-2-propen-1-one (4c): Colour: Light yellow crystals. FT-IR $\left(\mathrm{KBr}, v_{\max }, \mathrm{cm}^{-1}\right): 3127(\mathrm{~N}-\mathrm{H}), 3027(\mathrm{C}-\mathrm{H}$, aromatic), 2777 (C-H, aliphatic), $1703(\mathrm{C}=0), 1603$ (C=C, aliphatic), 1450 (C=C, aromatic), $688(\mathrm{C}-\mathrm{Cl}) .{ }^{1} \mathrm{H}$ NMR $(400 \mathrm{MHz}$, DMSO- $\left.d_{6}, \delta, \mathrm{ppm}\right): 2.41\left(\mathrm{~s}, 3 \mathrm{H}, \mathrm{CH}_{3}\right), 7.38-8.05(\mathrm{~m}, 8 \mathrm{H}, \mathrm{Ar}-\mathrm{H})$, $7.73(\mathrm{~d}, J=15.2 \mathrm{~Hz}, 1 \mathrm{H}, \mathrm{HC}=\mathrm{CH}(\mathrm{H}-\alpha)), 8.04(\mathrm{~d}, J=15.2 \mathrm{~Hz}, 1 \mathrm{H}$, $\mathrm{HC}=\mathrm{CH}(\mathrm{H}-\beta)), 9.69(\mathrm{~s}, 1 \mathrm{H}, \mathrm{NH})$. ESI-MS $(\mathrm{m} / \mathrm{z}): 386[\mathrm{M}+\mathrm{H}]^{+}$. 


\begin{tabular}{|c|c|c|c|c|c|c|c|c|c|c|c|c|}
\hline \multirow{3}{*}{ Compound } & \multirow[t]{3}{*}{$\mathbf{R}$} & \multirow{3}{*}{$\begin{array}{l}\text { Molecular } \\
\text { formula }\end{array}$} & \multirow{3}{*}{$\begin{array}{l}\text { Molecular } \\
\text { weight (g) }\end{array}$} & \multirow{3}{*}{$\begin{array}{l}\text { M.p. } \\
\left({ }^{\circ} \mathrm{C}\right)\end{array}$} & \multirow{3}{*}{$\begin{array}{l}\text { Yield } \\
(\%)^{a}\end{array}$} & \multicolumn{6}{|c|}{ \% Elemental analysis of $\mathrm{C}, \mathrm{H}, \mathrm{N}$ b } & \multirow{3}{*}{$\begin{array}{l}\text { MIC c } \\
(\mu \mathrm{g} / \mathrm{mL})\end{array}$} \\
\hline & & & & & & \multicolumn{3}{|c|}{ Calculated } & \multicolumn{3}{|c|}{ Found } & \\
\hline & & & & & & $\mathrm{C}$ & $\mathbf{H}$ & $\mathbf{N}$ & $\mathbf{C}$ & $\mathbf{H}$ & $\mathbf{N}$ & \\
\hline$\overline{4 a}$ & Phenyl & $\mathrm{C}_{18} \mathrm{H}_{12} \mathrm{Cl}_{2} \mathrm{~N}_{4} \mathrm{O}$ & 371 & 177 & 81 & 58.24 & 3.26 & 15.09 & 58.21 & 3.21 & 15.05 & 50 \\
\hline $4 b$ & $2-\mathrm{MeC}_{6} \mathrm{H}_{4}$ & $\mathrm{C}_{19} \mathrm{H}_{14} \mathrm{Cl}_{2} \mathrm{~N}_{4} \mathrm{O}$ & 385 & 165 & 79 & 59.24 & 3.66 & 14.54 & 59.22 & 3.62 & 14.52 & 25 \\
\hline $4 c$ & $3-\mathrm{MeC}_{6} \mathrm{H}_{4}$ & $\mathrm{C}_{19} \mathrm{H}_{14} \mathrm{Cl}_{2} \mathrm{~N}_{4} \mathrm{O}$ & 385 & 181 & 88 & 59.24 & 3.66 & 14.54 & 59.25 & 3.61 & 14.53 & 100 \\
\hline $4 d$ & $4-\mathrm{MeC}_{6} \mathrm{H}_{4}$ & $\mathrm{C}_{19} \mathrm{H}_{14} \mathrm{Cl}_{2} \mathrm{~N}_{4} \mathrm{O}$ & 385 & 144 & 75 & 59.24 & 3.66 & 14.54 & 59.22 & 3.64 & 14.51 & 50 \\
\hline $4 e$ & $2-\mathrm{OMeC}_{6} \mathrm{H}_{4}$ & $\mathrm{C}_{19} \mathrm{H}_{14} \mathrm{Cl}_{2} \mathrm{~N}_{4} \mathrm{O}_{2}$ & 401 & 152 & 91 & 56.87 & 3.52 & 13.96 & 56.82 & 3.51 & 13.95 & 6.25 \\
\hline $4 \mathrm{f}$ & $3-\mathrm{OMeC}_{6} \mathrm{H}_{4}$ & $\mathrm{C}_{19} \mathrm{H}_{14} \mathrm{Cl}_{2} \mathrm{~N}_{4} \mathrm{O}_{2}$ & 401 & 166 & 78 & 56.87 & 3.52 & 13.96 & 56.83 & 3.51 & 13.91 & 25 \\
\hline $4 \mathrm{~g}$ & $4-\mathrm{OMeC}_{6} \mathrm{H}_{4}$ & $\mathrm{C}_{19} \mathrm{H}_{14} \mathrm{Cl}_{2} \mathrm{~N}_{4} \mathrm{O}_{2}$ & 401 & 171 & 74 & 56.87 & 3.52 & 13.96 & 56.84 & 3.56 & 13.96 & 50 \\
\hline $4 \mathrm{~h}$ & $3-\mathrm{OHC}_{6} \mathrm{H}_{4}$ & $\mathrm{C}_{18} \mathrm{H}_{12} \mathrm{Cl}_{2} \mathrm{~N}_{4} \mathrm{O}_{2}$ & 387 & 118 & 77 & 55.83 & 3.12 & 14.47 & 55.85 & 3.11 & 14.42 & 50 \\
\hline $4 i$ & 4- $\mathrm{OHC}_{6} \mathrm{H}_{4}$ & $\mathrm{C}_{18} \mathrm{H}_{12} \mathrm{Cl}_{2} \mathrm{~N}_{4} \mathrm{O}_{2}$ & 387 & 129 & 88 & 55.83 & 3.12 & 14.47 & 55.83 & 3.11 & 14.45 & 100 \\
\hline $4 j$ & $3,5-\mathrm{diOHC}_{6} \mathrm{H}_{3}$ & $\mathrm{C}_{18} \mathrm{H}_{12} \mathrm{Cl}_{2} \mathrm{~N}_{4} \mathrm{O}_{3}$ & 403 & 147 & 85 & 53.62 & 3.00 & 13.89 & 53.61 & 3.02 & 13.81 & 50 \\
\hline $4 \mathrm{k}$ & $4,5-\mathrm{diOHC}_{6} \mathrm{H}_{3}$ & $\mathrm{C}_{18} \mathrm{H}_{12} \mathrm{Cl}_{2} \mathrm{~N}_{4} \mathrm{O}_{3}$ & 403 & 154 & 84 & 53.62 & 3.00 & 13.89 & 53.61 & 3.04 & 13.82 & 50 \\
\hline 41 & $2-\mathrm{Me}, 5-\mathrm{OHC}_{6} \mathrm{H}_{3}$ & $\mathrm{C}_{19} \mathrm{H}_{14} \mathrm{Cl}_{2} \mathrm{~N}_{4} \mathrm{O}_{2}$ & 401 & 169 & 85 & 56.87 & 3.52 & 13.96 & 56.86 & 3.51 & 13.93 & 25 \\
\hline $4 m$ & $2-\mathrm{NH}_{2} \mathrm{C}_{6} \mathrm{H}_{4}$ & $\mathrm{C}_{18} \mathrm{H}_{13} \mathrm{Cl}_{2} \mathrm{~N}_{5} \mathrm{O}$ & 386 & 179 & 83 & 55.97 & 3.39 & 18.13 & 55.95 & 3.31 & 18.11 & 50 \\
\hline $4 n$ & $3-\mathrm{NH}_{2} \mathrm{C}_{6} \mathrm{H}_{4}$ & $\mathrm{C}_{18} \mathrm{H}_{13} \mathrm{Cl}_{2} \mathrm{~N}_{5} \mathrm{O}$ & 386 & 122 & 81 & 55.97 & 3.39 & 18.13 & 55.94 & 3.32 & 18.12 & 100 \\
\hline 40 & $4-\mathrm{NH}_{2} \mathrm{C}_{6} \mathrm{H}_{4}$ & $\mathrm{C}_{18} \mathrm{H}_{13} \mathrm{Cl}_{2} \mathrm{~N}_{5} \mathrm{O}$ & 386 & 139 & 74 & 55.97 & 3.39 & 18.13 & 55.93 & 3.35 & 18.14 & 100 \\
\hline $4 p$ & $2-\mathrm{NO}_{2} \mathrm{C}_{6} \mathrm{H}_{4}$ & $\mathrm{C}_{18} \mathrm{H}_{11} \mathrm{Cl}_{2} \mathrm{~N}_{5} \mathrm{O}_{3}$ & 416 & 111 & 84 & 51.94 & 2.66 & 16.83 & 51.95 & 2.62 & 16.82 & 6.25 \\
\hline $4 q$ & $3-\mathrm{NO}_{2} \mathrm{C}_{6} \mathrm{H}_{4}$ & $\mathrm{C}_{18} \mathrm{H}_{11} \mathrm{Cl}_{2} \mathrm{~N}_{5} \mathrm{O}_{3}$ & 416 & 177 & 87 & 51.94 & 2.66 & 16.83 & 51.92 & 2.65 & 16.85 & 25 \\
\hline $4 \mathrm{r}$ & $4-\mathrm{NO}_{2} \mathrm{C}_{6} \mathrm{H}_{4}$ & $\mathrm{C}_{18} \mathrm{H}_{11} \mathrm{Cl}_{2} \mathrm{~N}_{5} \mathrm{O}_{3}$ & 416 & 174 & 74 & 51.94 & 2.66 & 16.83 & 51.93 & 2.62 & 16.81 & 50 \\
\hline $4 \mathrm{~s}$ & $2-\mathrm{ClC}_{6} \mathrm{H}_{4}$ & $\mathrm{C}_{18} \mathrm{H}_{11} \mathrm{Cl}_{3} \mathrm{~N}_{4} \mathrm{O}$ & 405 & 168 & 95 & 53.29 & 2.73 & 13.81 & 53.21 & 2.71 & 13.82 & 12.5 \\
\hline $4 t$ & $3-\mathrm{ClC}_{6} \mathrm{H}_{4}$ & $\mathrm{C}_{18} \mathrm{H}_{11} \mathrm{Cl}_{3} \mathrm{~N}_{4} \mathrm{O}$ & 405 & 151 & 77 & 53.29 & 2.73 & 13.81 & 53.22 & 2.74 & 13.81 & 100 \\
\hline $4 u$ & $4-\mathrm{ClC}_{6} \mathrm{H}_{4}$ & $\mathrm{C}_{18} \mathrm{H}_{11} \mathrm{Cl}_{3} \mathrm{~N}_{4} \mathrm{O}$ & 405 & 146 & 81 & 53.29 & 2.73 & 13.81 & 53.23 & 2.71 & 13.84 & 25 \\
\hline $4 v$ & 2,4- diClC $_{6} \mathrm{H}_{3}$ & $\mathrm{C}_{18} \mathrm{H}_{10} \mathrm{Cl}_{4} \mathrm{~N}_{4} \mathrm{O}$ & 440 & 194 & 92 & 49.12 & 2.29 & 12.73 & 49.11 & 2.25 & 12.71 & 50 \\
\hline $4 w$ & $2-\mathrm{FC}_{6} \mathrm{H}_{4}$ & $\mathrm{C}_{18} \mathrm{H}_{11} \mathrm{Cl}_{2} \mathrm{FN}_{4} \mathrm{O}$ & 389 & 112 & 97 & 55.55 & 2.85 & 14.39 & 55.53 & 2.82 & 14.35 & 50 \\
\hline $4 \mathrm{x}$ & $3-\mathrm{FC}_{6} \mathrm{H}_{4}$ & $\mathrm{C}_{18} \mathrm{H}_{11} \mathrm{Cl}_{2} \mathrm{FN}_{4} \mathrm{O}$ & 389 & 150 & 92 & 55.55 & 2.85 & 14.39 & 55.52 & 2.84 & 14.35 & 50 \\
\hline $4 y$ & $4-\mathrm{FC}_{6} \mathrm{H}_{4}$ & $\mathrm{C}_{18} \mathrm{H}_{11} \mathrm{Cl}_{2} \mathrm{FN}_{4} \mathrm{O}$ & 389 & 130 & 88 & 55.55 & 2.85 & 14.39 & 55.51 & 2.81 & 14.32 & 25 \\
\hline $4 \mathrm{z}$ & 2,4- $\mathrm{diFC}_{6} \mathrm{H}_{3}$ & $\mathrm{C}_{18} \mathrm{H}_{10} \mathrm{Cl}_{2} \mathrm{~F}_{2} \mathrm{~N}_{4} \mathrm{O}$ & 407 & 110 & 84 & 53.09 & 2.48 & 13.76 & 53.01 & 2.42 & 13.72 & 3.125 \\
\hline 4 аa & Furan-2yl & $\mathrm{C}_{16} \mathrm{H}_{10} \mathrm{Cl}_{2} \mathrm{~N}_{4} \mathrm{O}_{2}$ & 361 & 138 & 93 & 53.21 & 2.79 & 15.51 & 53.22 & 2.75 & 15.50 & 25 \\
\hline $4 b b$ & Thiophen-3-yl & $\mathrm{C}_{16} \mathrm{H}_{10} \mathrm{Cl}_{2} \mathrm{~N}_{4} \mathrm{OS}$ & 377 & 119 & 82 & 50.94 & 2.67 & 14.85 & 50.97 & 2.65 & 14.82 & 6.25 \\
\hline $4 c c$ & Pyrrol-2yl & $\mathrm{C}_{16} \mathrm{H}_{11} \mathrm{Cl}_{2} \mathrm{~N}_{5} \mathrm{O}$ & 360 & 122 & 85 & 66.25 & 3.42 & 11.89 & 66.22 & 3.41 & 11.86 & 25 \\
\hline $4 d d$ & Pyridin-2-yl & $\mathrm{C}_{17} \mathrm{H}_{11} \mathrm{Cl}_{2} \mathrm{~N}_{5} \mathrm{O}$ & 372 & 138 & 77 & 54.86 & 2.98 & 18.82 & 54.82 & 2.96 & 18.88 & 25 \\
\hline $4 \mathrm{ee}$ & Pyridin-3-yl & $\mathrm{C}_{17} \mathrm{H}_{11} \mathrm{Cl}_{2} \mathrm{~N}_{5} \mathrm{O}$ & 372 & 165 & 75 & 54.86 & 2.98 & 18.82 & 54.81 & 2.95 & 18.89 & 100 \\
\hline $4 \mathrm{ff}$ & Pyridin-4-yl & $\mathrm{C}_{17} \mathrm{H}_{11} \mathrm{Cl}_{2} \mathrm{~N}_{5} \mathrm{O}$ & 372 & 201 & 83 & 54.86 & 2.98 & 18.82 & 54.85 & 2.92 & 18.81 & 50 \\
\hline 4gg & Naphthalen-2-yl & $\mathrm{C}_{22} \mathrm{H}_{14} \mathrm{Cl}_{2} \mathrm{~N}_{4} \mathrm{O}$ & 421 & 119 & 87 & 62.72 & 3.35 & 13.30 & 62.71 & 3.32 & 13.32 & 100 \\
\hline $4 \mathrm{hh}$ & Naphthalen-3-yl & $\mathrm{C}_{22} \mathrm{H}_{14} \mathrm{Cl}_{2} \mathrm{~N}_{4} \mathrm{O}$ & 421 & 147 & 78 & 62.72 & 3.35 & 13.30 & 62.72 & 3.31 & 13.33 & 100 \\
\hline $4 \mathrm{ii}$ & Anthracen-9-yl & $\mathrm{C}_{26} \mathrm{H}_{16} \mathrm{Cl}_{2} \mathrm{~N}_{4} \mathrm{O}$ & 471 & 199 & 87 & 66.25 & 3.42 & 11.89 & 66.22 & 3.40 & 11.85 & 100 \\
\hline Ethambutol & & & & & & & & & & & & 3.125 \\
\hline Pyrazinamide & & & & & & & & & & & & 3.125 \\
\hline Streptomycin & & & & & & & & & & & & 6.25 \\
\hline
\end{tabular}
Streptomycin

a Crystallization solvent is ethanol

b Elemental analysis of $\mathrm{C}, \mathrm{H}$, and $\mathrm{N}$ were within $\pm 0.4 \%$ of theoretical value.

c Mycobacterium tuberculosis $\mathrm{H} 37 \mathrm{Rv}$.

(E)-1-(4-(4,6-Dichloro-1,3,5-triazin-2-ylamino)phenyl)-3-(4methylphenyl)-2-propen-1-one (4d): Colour: Light yellow crystals. FT-IR $\left(\mathrm{KBr}, v_{\max }, \mathrm{cm}^{-1}\right): 3122(\mathrm{~N}-\mathrm{H}), 3015(\mathrm{C}-\mathrm{H}$, aromatic), 2762 (C-H, aliphatic), $1705(\mathrm{C}=0), 1601$ (C=C, aliphatic), 1440 (C=C, aromatic), $685(\mathrm{C}-\mathrm{Cl}) .{ }^{1} \mathrm{H}$ NMR $(400 \mathrm{MHz}$, DMSO- $\left.d_{6}, \delta, \mathrm{ppm}\right): 2.39\left(\mathrm{~s}, 3 \mathrm{H}, \mathrm{CH}_{3}\right), 7.31-7.66(\mathrm{~m}, 8 \mathrm{H}, \mathrm{Ar}-\mathrm{H})$, $7.73(\mathrm{~d}, J=15.2 \mathrm{~Hz}, 1 \mathrm{H}, \mathrm{HC}=\mathrm{CH}(\mathrm{H}-\alpha)), 8.02(\mathrm{~d}, J=15.2 \mathrm{~Hz}, 1 \mathrm{H}$, $\mathrm{HC}=\mathrm{CH}(\mathrm{H}-\beta)), 9.62(\mathrm{~s}, 1 \mathrm{H}, \mathrm{NH})$. ESI-MS $(\mathrm{m} / \mathrm{z}): 386[\mathrm{M}+\mathrm{H}]^{+}$.

(E)-1-(4-(4,6-Dichloro-1,3,5-triazin-2-ylamino)phenyl)-3-(2methoxyphenyl)-2-propen-1-one (4e): Colour: Light yellow crystals. FT-IR (KBr, Vmax, cm-1): $3124(\mathrm{~N}-\mathrm{H}), 3027(\mathrm{C}-\mathrm{H}$, aromatic), 2975 (C-H, aliphatic), $1700(\mathrm{C}=0), 1603$ (C=C, aliphatic), 1417 (C=C, aromatic), $713(\mathrm{C}-\mathrm{Cl}), 1171(\mathrm{C}-\mathrm{O}-\mathrm{C})$, $1054(\mathrm{C}-0) .{ }^{1} \mathrm{H}$ NMR $\left(400 \mathrm{MHz}, \mathrm{DMSO}-d_{6}, \delta, \mathrm{ppm}\right): 3.86$ (s, $3 \mathrm{H}$, $\left.\mathrm{OCH}_{3}\right), 7.20-8.05(\mathrm{~m}, 8 \mathrm{H}, \mathrm{Ar}-\mathrm{H}), 7.48(\mathrm{~d}, J=15.2 \mathrm{~Hz}, 1 \mathrm{H}, \mathrm{HC}=\mathrm{CH}$ $(\mathrm{H}-\alpha)$ ), $8.05(\mathrm{~d}, J=15.2 \mathrm{~Hz}, 1 \mathrm{H}, \mathrm{HC}=\mathrm{CH}(\mathrm{H}-\beta)), 9.66(\mathrm{~s}, 1 \mathrm{H}, \mathrm{NH})$. ESI-MS $(\mathrm{m} / \mathrm{z}): 402[\mathrm{M}+\mathrm{H}]^{+}$.

(E)-1-(4-(4,6-Dichloro-1,3,5-triazin-2-ylamino)phenyl)-3-(3methoxyphenyl)-2-propen-1-one (4f): Colour: Light yellow crystals. FT-IR $\left(\mathrm{KBr}, v_{\max }, \mathrm{cm}^{-1}\right): 3124(\mathrm{~N}-\mathrm{H}), 3027(\mathrm{C}-\mathrm{H}$, aromatic), 2977 (C-H, aliphatic), $1700(\mathrm{C}=0), 1605(\mathrm{C}=\mathrm{C}$, aliphatic), $1457(\mathrm{C}=\mathrm{C}$, aromatic), $687(\mathrm{C}-\mathrm{Cl}), 1171(\mathrm{C}-\mathrm{O}-\mathrm{C})$, 1054 (C-0). ${ }^{1} \mathrm{H}$ NMR (400 MHz, DMSO- $\left.d_{6}, \delta, \mathrm{ppm}\right): 3.88$ (s, 3H, $\left.\mathrm{OCH}_{3}\right), 7.12-8.21(\mathrm{~m}, 8 \mathrm{H}, \mathrm{Ar}-\mathrm{H}), 7.71(\mathrm{~d}, J=15.2 \mathrm{~Hz}, 1 \mathrm{H}, \mathrm{HC}=\mathrm{CH}$ $(\mathrm{H}-\alpha)$ ), $8.06(\mathrm{~d}, J=15.2 \mathrm{~Hz}, 1 \mathrm{H}, \mathrm{HC}=\mathrm{CH}(\mathrm{H}-\beta)), 9.65(\mathrm{~s}, 1 \mathrm{H}, \mathrm{NH})$. ESI-MS $(m / z): 402[\mathrm{M}+\mathrm{H}]^{+}$.

(E)-1-(4-(4,6-Dichloro-1,3,5-triazin-2-ylamino)phenyl)-3-(4methoxyphenyl)-2-propen-1-one (4g): Colour: Light yellow crystals. FT-IR (KBr, $\left.v_{\max }, \mathrm{cm}^{-1}\right): 3122(\mathrm{~N}-\mathrm{H}), 3021(\mathrm{C}-\mathrm{H}$, aromatic), 2970 (C-H, aliphatic), 1690 (C=0), 1602 (C=C, aliphatic), 1455 (C=C, aromatic), $677(\mathrm{C}-\mathrm{Cl}), 1170(\mathrm{C}-\mathrm{O}-\mathrm{C})$, 1055 (C-0). ${ }^{1} \mathrm{H}$ NMR (400 MHz, DMSO-d6, $\left.\delta, \mathrm{ppm}\right): 3.86(\mathrm{~s}, 3 \mathrm{H}$, $\left.\mathrm{OCH}_{3}\right), 7.12-7.92(\mathrm{~m}, 8 \mathrm{H}, \mathrm{Ar}-\mathrm{H}), 7.71(\mathrm{~d}, J=15.2 \mathrm{~Hz}, 1 \mathrm{H}, \mathrm{HC}=\mathrm{CH}$ $(\mathrm{H}-\alpha)$ ), $8.05(\mathrm{~d}, J=15.2 \mathrm{~Hz}, 1 \mathrm{H}, \mathrm{HC}=\mathrm{CH}(\mathrm{H}-\beta)), 9.75(\mathrm{~s}, 1 \mathrm{H}, \mathrm{NH})$. ESI-MS $(m / z): 402[\mathrm{M}+\mathrm{H}]+$.

(E)-1-(4-(4,6-Dichloro-1,3,5-triazin-2-ylamino)phenyl)-3-(3hydroxyphenyl)-2-propen-1-one (4h): Colour: Light yellow crystals. FT-IR $\left(\mathrm{KBr}, v_{\max }, \mathrm{cm}^{-1}\right): 3445(\mathrm{O}-\mathrm{H}), 3124(\mathrm{~N}-\mathrm{H}), 3015$ (C-H, aromatic), 2984 (C-H, aliphatic), 1689 (C=0), 1606 (C=C, aliphatic), 1415 (C=C, aromatic), $676(\mathrm{C}-\mathrm{Cl}), 1054(\mathrm{C}-0) .{ }^{1} \mathrm{H}$ NMR $\left(400 \mathrm{MHz}, \mathrm{DMSO}-d_{6}, \delta, \mathrm{ppm}\right): 7.36-8.01(\mathrm{~m}, 8 \mathrm{H}, \mathrm{Ar}-\mathrm{H})$, $7.67(\mathrm{~d}, J=15.6 \mathrm{~Hz}, 1 \mathrm{H}, \mathrm{HC}=\mathrm{CH}(\mathrm{H}-\alpha)), 8.18(\mathrm{~d}, J=15.6 \mathrm{~Hz}, 1 \mathrm{H}$, $\mathrm{HC}=\mathrm{CH}(\mathrm{H}-\beta)), 9.85(\mathrm{~s}, 1 \mathrm{H}, \mathrm{NH}), 12.32(\mathrm{~s}, 1 \mathrm{H}, \mathrm{OH})$. ESI-MS $(\mathrm{m} / \mathrm{z}): 388[\mathrm{M}+\mathrm{H}]^{+}$.

(E)-1-(4-(4,6-Dichloro-1,3,5-triazin-2-ylamino)phenyl)-3-(4hydroxyphenyl)-2-propen-1-one (4i): Colour: Light yellow crystals. FT-IR $\left(\mathrm{KBr}, v_{\max }, \mathrm{cm}^{-1}\right): 3444(\mathrm{O}-\mathrm{H}), 3124(\mathrm{~N}-\mathrm{H}), 3019$ (C-H, aromatic), 2982 (C-H, aliphatic), $1684(\mathrm{C}=0), 1602$ (C=C, aliphatic), 1412 (C=C, aromatic), $671(\mathrm{C}-\mathrm{Cl}), 1055(\mathrm{C}-0) .{ }^{1} \mathrm{H}$ NMR $\left(400 \mathrm{MHz}, \mathrm{DMSO}-d_{6}, \delta, \mathrm{ppm}\right): 7.16-7.62(\mathrm{~m}, 8 \mathrm{H}, \mathrm{Ar}-\mathrm{H})$, $7.68(\mathrm{~d}, J=15.6 \mathrm{~Hz}, 1 \mathrm{H}, \mathrm{HC}=\mathrm{CH}(\mathrm{H}-\alpha)), 8.14(\mathrm{~d}, J=15.6 \mathrm{~Hz}, 1 \mathrm{H}$, $\mathrm{HC}=\mathrm{CH}(\mathrm{H}-\beta)), 9.82(\mathrm{~s}, 1 \mathrm{H}, \mathrm{NH}), 12.31(\mathrm{~s}, 1 \mathrm{H}, \mathrm{OH})$. ESI-MS $(\mathrm{m} / \mathrm{z}): 388[\mathrm{M}+\mathrm{H}]^{+}$.

(E)-1-(4-(4,6-dichloro-1,3,5-triazin-2-ylamino)phenyl)-3-(3, 5-dihydroxyphenyl)-2-propen-1-one (4j): Colour: Light yellow crystals. FT-IR $\left(\mathrm{KBr}, v_{\max }, \mathrm{cm}^{-1}\right): 3440(\mathrm{O}-\mathrm{H}), 3122(\mathrm{~N}-\mathrm{H}), 3027$ (C-H, aromatic), 2890 (C-H, aliphatic), 1700 (C=0), 1605 (C=C, aliphatic), 1511 (C=C, aromatic), $688(\mathrm{C}-\mathrm{Cl}), 1054(\mathrm{C}-0) .{ }^{1} \mathrm{H}$ NMR (400 MHz, DMSO- $\left.d_{6}, \delta, \mathrm{ppm}\right):$ 7.21-8.02 (m, 7H, Ar-H), $7.79(\mathrm{~d}, J=15.3 \mathrm{~Hz}, 1 \mathrm{H}, \mathrm{HC}=\mathrm{CH}(\mathrm{H}-\alpha)), 8.03(\mathrm{~d}, J=15.3 \mathrm{~Hz}, 1 \mathrm{H}$, 
$\mathrm{HC}=\mathrm{CH}(\mathrm{H}-\beta)), 9.89(\mathrm{~s}, 1 \mathrm{H}, \mathrm{NH}), 11.52$ (s, 2H, OH). ESI-MS $(\mathrm{m} / \mathrm{z}): 404[\mathrm{M}+\mathrm{H}]^{+}$.

(E)-1-(4-(4,6-Dichloro-1,3,5-triazin-2-ylamino)phenyl)-3-(4,5 -dihydroxyphenyl)-2-propen-1-one (4k): Colour: Light yellow crystals. FT-IR $\left(\mathrm{KBr}, v_{\max }, \mathrm{cm}^{-1}\right): 3395(\mathrm{O}-\mathrm{H}), 3127(\mathrm{~N}-\mathrm{H}), 3017$ (C-H, aromatic), 2989 (C-H, aliphatic), 1686 (C=0), 1615 (C=C, aliphatic), 1545 (C=C, aromatic), 689 (C-Cl), $1054(\mathrm{C}-0) .{ }^{1} \mathrm{H}$ NMR (400 MHz, DMSO- $\left.d_{6}, \delta, p p m\right): 7.55-8.03(\mathrm{~m}, 7 \mathrm{H}, \mathrm{Ar}-\mathrm{H})$, $7.83(\mathrm{~d}, J=15.3 \mathrm{~Hz}, 1 \mathrm{H}, \mathrm{HC}=\mathrm{CH}(\mathrm{H}-\alpha)), 8.08(\mathrm{~d}, J=15.3 \mathrm{~Hz}, 1 \mathrm{H}$, $\mathrm{HC}=\mathrm{CH}(\mathrm{H}-\beta)$ ), $9.58(\mathrm{~s}, 1 \mathrm{H}, \mathrm{OH}), 9.87$ (s, 1H, NH), 10.57 (s, 1H, OH). ESI-MS $(m / z): 404[\mathrm{M}+\mathrm{H}]+$.

(E)-1-(4-(4,6-Dichloro-1,3,5-triazin-2-ylamino)phenyl)-3-(2methyl-5-hydroxyphenyl)-2-propen-1-one (41): Colour: Light yellow crystals. FT-IR $\left(\mathrm{KBr}, v_{\max }, \mathrm{cm}^{-1}\right): 3440(\mathrm{O}-\mathrm{H}), 3122$ $(\mathrm{N}-\mathrm{H}), 3021$ (C-H, aromatic), $2975(\mathrm{C}-\mathrm{H}$, aliphatic), 1690 $(\mathrm{C}=0), 1641$ ( $\mathrm{C}=\mathrm{C}$, aliphatic), 1486 ( $\mathrm{C}=\mathrm{C}$, aromatic), $678(\mathrm{C}-\mathrm{Cl})$, $1054(\mathrm{C}-0) .{ }^{1} \mathrm{H}$ NMR $\left(400 \mathrm{MHz}, \mathrm{DMSO}-d_{6}, \delta, \mathrm{ppm}\right): 2.47$ (s, 3H, $\left.\mathrm{CH}_{3}\right), 7.62-8.01(\mathrm{~m}, 7 \mathrm{H}, \mathrm{Ar}-\mathrm{H}), 7.81(\mathrm{~d}, J=15.3 \mathrm{~Hz}, 1 \mathrm{H}, \mathrm{HC}=\mathrm{CH}$ $(\mathrm{H}-\alpha)$ ), 8.08 (d, $J=15.3 \mathrm{~Hz}, 1 \mathrm{H}, \mathrm{HC}=\mathrm{CH}(\mathrm{H}-\beta)), 9.01(\mathrm{~s}, 1 \mathrm{H}, \mathrm{NH})$, $10.52(\mathrm{~s}, 1 \mathrm{H}, \mathrm{OH})$. ESI-MS $(\mathrm{m} / \mathrm{z}): 402[\mathrm{M}+\mathrm{H}]^{+}$.

(E)-1-(4-(4,6-Dichloro-1,3,5-triazin-2-ylamino)phenyl)-3-(2aminophenyl)-2-propen-1-one (4m): Colour: Light yellow crystals. FT-IR $\left(\mathrm{KBr}, v_{\max }, \mathrm{cm}^{-1}\right): 3367\left(\mathrm{NH}_{2}\right), 3117(\mathrm{~N}-\mathrm{H}), 2978$ (C-H, aromatic), 2763 (C-H, aliphatic), 1693 (C=0), 1597 (C=C, aliphatic), 1413 (C=C, aromatic), 688 (C-Cl), $1296(\mathrm{C}-\mathrm{N}) .{ }^{1} \mathrm{H}$ NMR (400 MHz, DMSO- $\left.d_{6}, \delta, p p m\right): 7.74-8.11(\mathrm{~m}, 8 \mathrm{H}, \mathrm{Ar}-\mathrm{H})$, $7.58(\mathrm{~d}, J=15.2 \mathrm{~Hz}, 1 \mathrm{H}, \mathrm{HC}=\mathrm{CH}(\mathrm{H}-\alpha)), 8.06(\mathrm{~d}, J=15.2 \mathrm{~Hz}, 1 \mathrm{H}$, $\mathrm{HC}=\mathrm{CH}(\mathrm{H}-\beta)$ ), 9.65 (s, 1H, NH), 10.51 (s, 2H, Ar-NH2). ESI-MS $(\mathrm{m} / \mathrm{z}): 387[\mathrm{M}+\mathrm{H}]^{+}$.

(E)-1-(4-(4,6-Dichloro-1,3,5-triazin-2-ylamino)phenyl)-3-(3aminophenyl)-2-propen-1-one (4n): Colour: Light yellow crystals. FT-IR (KBr, $\left.v_{\max }, \mathrm{cm}^{-1}\right): 3367\left(\mathrm{NH}_{2}\right), 3117(\mathrm{~N}-\mathrm{H}), 2978$ (C-H, aromatic), 2763 (C-H, aliphatic), $1693(\mathrm{C}=0), 1597$ (C=C, aliphatic), $1413\left(\mathrm{C}=\mathrm{C}\right.$, aromatic), $688(\mathrm{C}-\mathrm{Cl}), 1290(\mathrm{C}-\mathrm{N}) .{ }^{1} \mathrm{H}$ NMR $\left(400 \mathrm{MHz}\right.$, DMSO- $\left.d_{6}, \delta, \mathrm{ppm}\right): 7.72(\mathrm{~d}, J=15.2 \mathrm{~Hz}, 1 \mathrm{H}$, $\mathrm{HC}=\mathrm{CH}(\mathrm{H}-\alpha)), 7.74-8.11(\mathrm{~m}, 8 \mathrm{H}, \mathrm{Ar}-\mathrm{H}), 8.01(\mathrm{~d}, J=15.2 \mathrm{~Hz}, 1 \mathrm{H}$, $\mathrm{HC}=\mathrm{CH}(\mathrm{H}-\beta)), 9.67(\mathrm{~s}, 1 \mathrm{H}, \mathrm{NH}), 10.54\left(\mathrm{~s}, 2 \mathrm{H}, \mathrm{Ar}-\mathrm{NH}_{2}\right)$. ESI-MS $(\mathrm{m} / \mathrm{z}): 387[\mathrm{M}+\mathrm{H}]^{+}$.

(E)-1-(4-(4,6-Dichloro-1,3,5-triazin-2-ylamino)phenyl)-3-(4aminophenyl)-2-propen-1-one (40): Colour: Light yellow crystals. FT-IR $\left(\mathrm{KBr}, v_{\max }, \mathrm{cm}^{-1}\right): 3362\left(\mathrm{NH}_{2}\right), 3115(\mathrm{~N}-\mathrm{H}), 2979$ (C-H, aromatic), 2761 (C-H, aliphatic), $1690(\mathrm{C}=0), 1590(\mathrm{C}=\mathrm{C}$, aliphatic), 1410 (C=C, aromatic), 684 (C-Cl), $1290(\mathrm{C}-\mathrm{N}) .{ }^{1} \mathrm{H}$ NMR (400 MHz, DMSO- $\left.d_{6}, \delta, \mathrm{ppm}\right): 7.71(\mathrm{~d}, J=15.2 \mathrm{~Hz}, 1 \mathrm{H}$, $\mathrm{HC}=\mathrm{CH}(\mathrm{H}-\alpha)), 7.77-8.14(\mathrm{~m}, 8 \mathrm{H}, \mathrm{Ar}-\mathrm{H}), 8.12(\mathrm{~d}, J=15.2 \mathrm{~Hz}, 1 \mathrm{H}$, $\mathrm{HC}=\mathrm{CH}(\mathrm{H}-\beta)$ ), 9.65 (s, 1H, NH), 10.52 (s, 2H, Ar-NH2). ESI-MS $(\mathrm{m} / \mathrm{z}): 387[\mathrm{M}+\mathrm{H}]^{+}$.

(E)-1-(4-(4,6-Dichloro-1,3,5-triazin-2-ylamino)phenyl)-3-(2nitrophenyl)-2-propen-1-one (4p): Colour: Light yellow crystals. FT-IR (KBr, $\left.v_{\max }, \mathrm{cm}^{-1}\right): 3122(\mathrm{~N}-\mathrm{H}), 3024(\mathrm{C}-\mathrm{H}$, aromatic), $2776(\mathrm{C}-\mathrm{H}$, aliphatic), $1700(\mathrm{C}=0), 1604$ ( $\mathrm{C}=\mathrm{C}$, aliphatic), 1414 (C=C, aromatic), $688(\mathrm{C}-\mathrm{Cl}), 1529(\mathrm{~N}=0), 1291(\mathrm{C}-\mathrm{N}) .{ }^{1} \mathrm{H}$ NMR (400 MHz, DMSO- $\left.d_{6}, \delta, p p m\right): 6.86-8.18(\mathrm{~m}, 8 \mathrm{H}, \mathrm{Ar}-\mathrm{H}), 8.05$ (d, $J$ $=15.2 \mathrm{~Hz}, 1 \mathrm{H}, \mathrm{HC}=\mathrm{CH}(\mathrm{H}-\alpha)), 8.35(\mathrm{~d}, J=15.2 \mathrm{~Hz}, 1 \mathrm{H}, \mathrm{HC}=\mathrm{CH}$ (H- $\beta)), 9.72(\mathrm{~s}, 1 \mathrm{H}, \mathrm{NH})$. ESI-MS $(\mathrm{m} / \mathrm{z}): 417[\mathrm{M}+\mathrm{H}]^{+}$.

(E)-1-(4-(4,6-Dichloro-1,3,5-triazin-2-ylamino)phenyl)-3-(3nitrophenyl)-2-propen-1-one (4q): Colour: Light yellow crystals. FT-IR (KBr, $\left.v_{\max }, \mathrm{cm}^{-1}\right)$ : $3115(\mathrm{~N}-\mathrm{H}), 3026(\mathrm{C}-\mathrm{H}$, aromatic), 2775 (C-H, aliphatic), 1700 (C=0), 1599 (C=C, aliphatic), 1412 $\left(\mathrm{C}=\mathrm{C}\right.$, aromatic), $688(\mathrm{C}-\mathrm{Cl}), 1522(\mathrm{~N}=0), 1290(\mathrm{C}-\mathrm{N}) .{ }^{1} \mathrm{H}$ NMR (400 MHz, DMSO- $\left.d_{6}, \delta, \mathrm{ppm}\right): 7.55-8.39(\mathrm{~m}, 8 \mathrm{H}, \mathrm{Ar}-\mathrm{H}), 7.86(\mathrm{~d}, J$ $=15.2 \mathrm{~Hz}, 1 \mathrm{H}, \mathrm{HC}=\mathrm{CH}(\mathrm{H}-\alpha)), 8.06(\mathrm{~d}, J=15.2 \mathrm{~Hz}, 1 \mathrm{H}, \mathrm{HC}=\mathrm{CH}$ $(\mathrm{H}-\beta)), 9.73(\mathrm{~s}, 1 \mathrm{H}, \mathrm{NH})$. ESI-MS $(\mathrm{m} / \mathrm{z}): 417[\mathrm{M}+\mathrm{H}]^{+}$.

(E)-1-(4-(4,6-Dichloro-1,3,5-triazin-2-ylamino)phenyl)-3-(4nitrophenyl)-2-propen-1-one (4r): Colour: Light yellow crystals. FT-IR (KBr, $\left.v_{\max }, \mathrm{cm}^{-1}\right): 3205(\mathrm{~N}-\mathrm{H}), 3016(\mathrm{C}-\mathrm{H}$, aromatic), 2895 (C-H, aliphatic), 1710 (C=0), 1589 (C=C, aliphatic), 1442 $\left(\mathrm{C}=\mathrm{C}\right.$, aromatic), $680(\mathrm{C}-\mathrm{Cl}), 1520(\mathrm{~N}=0), 1287(\mathrm{C}-\mathrm{N}) .{ }^{1} \mathrm{H}$ NMR (400 MHz, DMSO- $\left.d_{6}, \delta, \mathrm{ppm}\right)$ : 7.54-8.29 (m, 8H, Ar-H), $7.83(\mathrm{~d}, J$ $=15.2 \mathrm{~Hz}, 1 \mathrm{H}, \mathrm{HC}=\mathrm{CH}(\mathrm{H}-\alpha)$ ), $8.07(\mathrm{~d}, J=15.2 \mathrm{~Hz}, 1 \mathrm{H}, \mathrm{HC}=\mathrm{CH}$

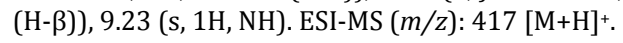

(E)-1-(4-(4,6-Dichloro-1,3,5-triazin-2-ylamino)phenyl)-3-(2chlorophenyl)-2-propen-1-one (4s): Colour: Light yellow crystals. FT-IR $\left(\mathrm{KBr}, v_{\max } \mathrm{cm}^{-1}\right): 3127(\mathrm{~N}-\mathrm{H}), 3027(\mathrm{C}-\mathrm{H}$, aromatic), 2893 (C-H, aliphatic), 1689 (C=0), 1597 (C=C, aliphatic), $1450\left(\mathrm{C}=\mathrm{C}\right.$, aromatic), $688(\mathrm{C}-\mathrm{Cl}), 786(\mathrm{C}-\mathrm{Cl}) .{ }^{1} \mathrm{H}$ NMR (400 MHz, DMSO-d6, $\delta, p p m): 7.60(\mathrm{~d}, J=15.2 \mathrm{~Hz}, 1 \mathrm{H}$, $\mathrm{HC}=\mathrm{CH}(\mathrm{H}-\alpha)), 7.62-8.24(\mathrm{~m}, 8 \mathrm{H}, \mathrm{Ar}-\mathrm{H}), 7.78(\mathrm{~d}, J=15.2 \mathrm{~Hz}, 1 \mathrm{H}$, $\mathrm{HC}=\mathrm{CH}(\mathrm{H}-\beta)), 9.65(\mathrm{~s}, 1 \mathrm{H}, \mathrm{NH})$. ESI-MS $(\mathrm{m} / \mathrm{z}): 406[\mathrm{M}+\mathrm{H}]^{+}$.

(E)-1-(4-(4,6-Dichloro-1,3,5-triazin-2-ylamino)phenyl)-3-(3chlorophenyl)-2-propen-1-one (4t): Colour: Light yellow crystals. FT-IR $\left(\mathrm{KBr}, v_{\max } \mathrm{cm}^{-1}\right): 3121(\mathrm{~N}-\mathrm{H}), 3025(\mathrm{C}-\mathrm{H}$ aromatic), 2891 (C-H, aliphatic), 1686 (C=0), 1594 (C=C, aliphatic), 1451 (C=C, aromatic), $786(\mathrm{C}-\mathrm{Cl}) .{ }^{1} \mathrm{H}$ NMR $(400 \mathrm{MHz}$, DMSO- $\left.d_{6}, \delta, \mathrm{ppm}\right): 7.45(\mathrm{~d}, J=15.2 \mathrm{~Hz}, 1 \mathrm{H}, \mathrm{HC}=\mathrm{CH}(\mathrm{H}-\alpha)), 7.62$ $7.74(\mathrm{~m}, 8 \mathrm{H}, \mathrm{Ar}-\mathrm{H}), 7.79(\mathrm{~d}, J=15.2 \mathrm{~Hz}, 1 \mathrm{H}, \mathrm{HC}=\mathrm{CH}(\mathrm{H}-\beta)), 9.65$ (s, $1 \mathrm{H}, \mathrm{NH})$. ESI-MS $(\mathrm{m} / \mathrm{z}): 406[\mathrm{M}+\mathrm{H}]^{+}$.

(E)-1-(4-(4,6-Dichloro-1,3,5-triazin-2-ylamino)phenyl)-3-(4chlorophenyl)-2-propen-1-one (4u): Colour: Light yellow crystals. FT-IR $\left(\mathrm{KBr}, v_{\max } \mathrm{cm}^{-1}\right): 3126(\mathrm{~N}-\mathrm{H}), 3023(\mathrm{C}-\mathrm{H}$, aromatic), 2883 (C-H, aliphatic), $1690(\mathrm{C}=0), 1588$ (C=C, aliphatic), 1442 (C=C, aromatic), $681(\mathrm{C}-\mathrm{Cl}), 785(\mathrm{C}-\mathrm{Cl}) .{ }^{1} \mathrm{H}$ NMR $\left(400 \mathrm{MHz}, \mathrm{DMSO}-d_{6}, \delta, \mathrm{ppm}\right): 7.61(\mathrm{~d}, J=15.2 \mathrm{~Hz}, 1 \mathrm{H}$, $\mathrm{HC}=\mathrm{CH}(\mathrm{H}-\alpha)), 7.67-7.82(\mathrm{~m}, 8 \mathrm{H}, \mathrm{Ar}-\mathrm{H}), 7.87(\mathrm{~d}, J=15.2 \mathrm{~Hz}, 1 \mathrm{H}$, $\mathrm{HC}=\mathrm{CH}(\mathrm{H}-\beta)), 9.63(\mathrm{~s}, 1 \mathrm{H}, \mathrm{NH})$. ESI-MS $(\mathrm{m} / \mathrm{z}): 406[\mathrm{M}+\mathrm{H}]^{+}$.

(E)-1-(4-(4,6-Dichloro-1,3,5-triazin-2-ylamino)phenyl)-3-(2, 4-dichlorophenyl)-2-propen-1-one (4v): Colour: Light yellow crystals. FT-IR (KBr, $\left.v_{\max }, \mathrm{cm}^{-1}\right): 3124(\mathrm{~N}-\mathrm{H}), 3018(\mathrm{C}-\mathrm{H}$, aromatic), 2891 (C-H, aliphatic), 1689 (C=0), 1641 (C=C, aliphatic), $1485\left(\mathrm{C}=\mathrm{C}\right.$, aromatic), $691(\mathrm{C}-\mathrm{Cl}), 786(\mathrm{C}-\mathrm{Cl}) .{ }^{1} \mathrm{H}$ NMR (400 MHz, DMSO-d6, $\delta, p p m): 7.65-8.23(\mathrm{~m}, 7 \mathrm{H}, \mathrm{Ar}-\mathrm{H})$, $7.78(\mathrm{~d}, J=15.2 \mathrm{~Hz}, 1 \mathrm{H}, \mathrm{HC}=\mathrm{CH}(\mathrm{H}-\alpha)), 8.06(\mathrm{~d}, J=15.2 \mathrm{~Hz}, 1 \mathrm{H}$, $\mathrm{HC}=\mathrm{CH}(\mathrm{H}-\beta)), 9.69(\mathrm{~s}, 1 \mathrm{H}, \mathrm{NH})$. ESI-MS $(\mathrm{m} / \mathrm{z}): 441[\mathrm{M}+\mathrm{H}]^{+}$.

(E)-1-(4-(4,6-Dichloro-1,3,5-triazin-2-ylamino)phenyl)-3-(2fluorophenyl)-2-propen-1-one (4w): Colour: Light yellow crystals. FT-IR (KBr, $\left.v_{\max } \mathrm{cm}^{-1}\right): 3117(\mathrm{~N}-\mathrm{H}), 3017(\mathrm{C}-\mathrm{H}$, aromatic), 2977 (C-H, aliphatic), 1693 (C=0), 1605 (C=C, aliphatic), 1415 (C=C, aromatic), $688(\mathrm{C}-\mathrm{Cl}), 1116(\mathrm{C}-\mathrm{F}) .{ }^{1} \mathrm{H}$ NMR (400 MHz, DMSO- $\left.d_{6}, \delta, \mathrm{ppm}\right): 7.36-8.03(\mathrm{~m}, 8 \mathrm{H}, \mathrm{Ar}-\mathrm{H})$ $7.55(\mathrm{~d}, J=15.2 \mathrm{~Hz}, 1 \mathrm{H}, \mathrm{HC}=\mathrm{CH}(\mathrm{H}-\alpha)), 7.82(\mathrm{~d}, J=15.2 \mathrm{~Hz}, 1 \mathrm{H}$, $\mathrm{HC}=\mathrm{CH}(\mathrm{H}-\beta)), 9.68(\mathrm{~s}, 1 \mathrm{H}, \mathrm{NH})$. ESI-MS $(\mathrm{m} / \mathrm{z}): 390[\mathrm{M}+\mathrm{H}]^{+}$.

(E)-1-(4-(4,6-Dichloro-1,3,5-triazin-2-ylamino)phenyl)-3-(3fluorophenyl)-2-propen-1-one (4x): Colour: Light yellow crystals. FT-IR (KBr, $\left.v_{\max }, \mathrm{cm}^{-1}\right): 3112(\mathrm{~N}-\mathrm{H}), 3011(\mathrm{C}-\mathrm{H}$ aromatic), 2974 (C-H, aliphatic), 1690 (C=0), 1602 (C=C, aliphatic), 1412 (C=C, aromatic), $680(\mathrm{C}-\mathrm{Cl}), 1011(\mathrm{C}-\mathrm{F}) .{ }^{1} \mathrm{H}$ NMR $\left(400 \mathrm{MHz}\right.$, DMSO- $\left.d_{6}, \delta, \mathrm{ppm}\right):$ 7.16-7.73 (m, 8H, Ar-H), $7.75(\mathrm{~d}, J=15.2 \mathrm{~Hz}, 1 \mathrm{H}, \mathrm{HC}=\mathrm{CH}(\mathrm{H}-\alpha)), 7.81(\mathrm{~d}, J=15.2 \mathrm{~Hz}, 1 \mathrm{H}$, $\mathrm{HC}=\mathrm{CH}(\mathrm{H}-\beta)), 9.78(\mathrm{~s}, 1 \mathrm{H}, \mathrm{NH})$. ESI-MS $(\mathrm{m} / \mathrm{z}): 390[\mathrm{M}+\mathrm{H}]^{+}$.

(E)-1-(4-(4,6-Dichloro-1,3,5-triazin-2-ylamino)phenyl)-3-(4fluorophenyl)-2-propen-1-one (4y): Colour: Light yellow crystals. FT-IR $\left(\mathrm{KBr}, v_{\max } \mathrm{cm}^{-1}\right): 3114(\mathrm{~N}-\mathrm{H}), 3212(\mathrm{C}-\mathrm{H}$, aromatic), 2975 (C-H, aliphatic), 1694 (C=0), 1602 (C=C, aliphatic), 1412 (C=C, aromatic), $1106(\mathrm{C}-\mathrm{F}), 685(\mathrm{C}-\mathrm{Cl}) .{ }^{1} \mathrm{H}$ NMR (400 MHz, DMSO- $\left.d_{6}, \delta, \mathrm{ppm}\right): 7.22-7.63(\mathrm{~m}, 8 \mathrm{H}, \mathrm{Ar}-\mathrm{H})$, $7.65(\mathrm{~d}, J=15.2 \mathrm{~Hz}, 1 \mathrm{H}, \mathrm{HC}=\mathrm{CH}(\mathrm{H}-\alpha)), 7.82(\mathrm{~d}, J=15.2 \mathrm{~Hz}, 1 \mathrm{H}$, $\mathrm{HC}=\mathrm{CH}(\mathrm{H}-\beta)), 9.77(\mathrm{~s}, 1 \mathrm{H}, \mathrm{NH})$. ESI-MS $(\mathrm{m} / \mathrm{z}): 390[\mathrm{M}+\mathrm{H}]^{+}$.

(E)-1-(4-(4,6-Dichloro-1,3,5-triazin-2-ylamino)phenyl)-3-(2, 4-difluorophenyl)-2-propen-1-one (4z): Colour: Light yellow crystals. FT-IR (KBr, $\left.V_{\max } \mathrm{cm}^{-1}\right): 3122(\mathrm{~N}-\mathrm{H}), 3021(\mathrm{C}-\mathrm{H}$, aromatic), 2884 (C-H, aliphatic), 1693 (C=0), 1605 (C=C, aliphatic), 1415 (C=C, aromatic), $688(\mathrm{C}-\mathrm{Cl}), 1114(\mathrm{C}-\mathrm{F}) .{ }^{1} \mathrm{H}$ NMR (400 MHz, DMSO- $\left.d_{6}, \delta, p p m\right): 7.39-8.31(\mathrm{~m}, 7 \mathrm{H}, \mathrm{Ar}-\mathrm{H})$, $7.76(\mathrm{~d}, J=15.2 \mathrm{~Hz}, 1 \mathrm{H}, \mathrm{HC}=\mathrm{CH}(\mathrm{H}-\alpha)), 8.08(\mathrm{~d}, J=15.2 \mathrm{~Hz}, 1 \mathrm{H}$, $\mathrm{HC}=\mathrm{CH}(\mathrm{H}-\beta)), 9.69(\mathrm{~s}, 1 \mathrm{H}, \mathrm{NH})$. ESI-MS $(\mathrm{m} / \mathrm{z}): 408[\mathrm{M}+\mathrm{H}]^{+}$.

(E)-1-(4-(4,6-Dichloro-1,3,5-triazin-2-ylamino)phenyl)-3(furan-2-yl)-2-propen-1-one (4aa): Colour: Light yellow crystals. FT-IR $\left(\mathrm{KBr}, v_{\max }, \mathrm{cm}^{-1}\right): 3420(\mathrm{~N}-\mathrm{H}), 3062(\mathrm{C}-\mathrm{H}$, aromatic), 3030 (C-H, aliphatic), 1671(C=0), 1591 (C=C, aliphatic), 1453 (C=C, aromatic), $696(\mathrm{C}-\mathrm{Cl}), 1155(\mathrm{C}-\mathrm{O}-\mathrm{C})$, $1053(\mathrm{C}-0) .{ }^{1} \mathrm{H}$ NMR $\left(400 \mathrm{MHz}, \mathrm{DMSO}-d_{6}, \delta, \mathrm{ppm}\right): 6.74(\mathrm{~s}, 1 \mathrm{H}$, Ar-H), 6.21 (m, 1H, Ar-H), 7.16-7.50 (m, 5H, Ar-H), 7.62 (d, J = 
$16 \mathrm{~Hz}, 1 \mathrm{H}, \mathrm{HC}=\mathrm{CH}(\mathrm{H}-\alpha)), 8.06(\mathrm{~d}, J=16 \mathrm{~Hz}, 1 \mathrm{H}, \mathrm{HC}=\mathrm{CH}(\mathrm{H}-\beta))$, $9.73(\mathrm{~s}, 1 \mathrm{H}, \mathrm{NH})$. ESI-MS $(\mathrm{m} / \mathrm{z}): 362[\mathrm{M}+\mathrm{H}]^{+}$.

(E)-1-(4-(4,6-Dichloro-1,3,5-triazin-2-ylamino)phenyl)-3(thiophen-3-yl)-2-propen-1-one (4bb): Colour: Light yellow crystals. FT-IR $\left(\mathrm{KBr}, v_{\max }, \mathrm{cm}^{-1}\right): 3430(\mathrm{~N}-\mathrm{H}), 3019(\mathrm{C}-\mathrm{H}$, aromatic), 2973 (C-H, aliphatic), 1689 (C=0), 1599 (C=C, aliphatic), $1414\left(\mathrm{C}=\mathrm{C}\right.$, aromatic), $688(\mathrm{C}-\mathrm{Cl}) .{ }^{1} \mathrm{H}$ NMR $(400 \mathrm{MHz}$, DMSO- $\left.d_{6}, \delta, \mathrm{ppm}\right): 6.68(\mathrm{~s}, 1 \mathrm{H}, \mathrm{Ar}-\mathrm{H}), 6.91(\mathrm{~s}, 1 \mathrm{H}, \mathrm{Ar}-\mathrm{H}), 7.12(\mathrm{~s}$, $1 \mathrm{H}, \mathrm{Ar}-\mathrm{H}), 7.33-7.58(\mathrm{~m}, 4 \mathrm{H}, \mathrm{Ar}-\mathrm{H}), 7.76(\mathrm{~d}, J=15.2 \mathrm{~Hz}, 1 \mathrm{H}$, $\mathrm{HC}=\mathrm{CH}(\mathrm{H}-\alpha)$ ), $8.02(\mathrm{~d}, J=15.2 \mathrm{~Hz}, 1 \mathrm{H}, \mathrm{HC}=\mathrm{CH}(\mathrm{H}-\beta)), 9.68(\mathrm{~s}$, $1 \mathrm{H}, \mathrm{NH})$. ESI-MS $(\mathrm{m} / \mathrm{z}): 378[\mathrm{M}+\mathrm{H}]^{+}$.

(E)-1-(4-(4,6-Dichloro-1,3,5-triazin-2-ylamino)phenyl)-3(pyrrol-2-yl)-2-propen-1-one (4cc): Colour: Light yellow crystals. FT-IR $\left(\mathrm{KBr}, v_{\max }, \mathrm{cm}^{-1}\right): 3144(\mathrm{~N}-\mathrm{H}), 3052(\mathrm{~N}-\mathrm{H}), 3017$ (C-H, aromatic), 2973 (C-H, aliphatic), $1695(\mathrm{C}=0), 1615(\mathrm{C}=\mathrm{C}$, aliphatic), $1414\left(\mathrm{C}=\mathrm{C}\right.$, aromatic), $678(\mathrm{C}-\mathrm{Cl}), 1308(\mathrm{C}-\mathrm{N}) .{ }^{1} \mathrm{H}$ NMR $\left(400 \mathrm{MHz}\right.$, DMSO- $\left.d_{6}, \delta, \mathrm{ppm}\right): 6.46(\mathrm{~s}, 1 \mathrm{H}, \mathrm{Ar}-\mathrm{H}), 7.44(\mathrm{~m}$, $1 \mathrm{H}, \mathrm{Ar}-\mathrm{H}), 7.55-7.61(\mathrm{~m}, 5 \mathrm{H}, \mathrm{Ar}-\mathrm{H}), 7.76(\mathrm{~d}, J=15.2 \mathrm{~Hz}, 1 \mathrm{H}$, $\mathrm{HC}=\mathrm{CH}(\mathrm{H}-\alpha)$ ), $8.03(\mathrm{~d}, J=15.2 \mathrm{~Hz}, 1 \mathrm{H}, \mathrm{HC}=\mathrm{CH}(\mathrm{H}-\beta)), 9.64(\mathrm{~s}$, $1 \mathrm{H}, \mathrm{NH}), 10.55(\mathrm{~s}, 1 \mathrm{H}, \mathrm{NH})$. ESI-MS $(\mathrm{m} / \mathrm{z}): 361[\mathrm{M}+\mathrm{H}]^{+}$.

(E)-1-(4-(4,6-Dichloro-1,3,5-triazin-2-ylamino)phenyl)-3(pyridin-2-yl)-2-propen-1-one (4dd): Colour: Light yellow crystals. FT-IR $\left(\mathrm{KBr}, v_{\max } \mathrm{cm}^{-1}\right): 3127(\mathrm{~N}-\mathrm{H}), 3019(\mathrm{C}-\mathrm{H}$, aromatic), 2931 (C-H, aliphatic), 1689 (C=0), 1604 (C=C, aliphatic), 1417 (C=C, aromatic), $688(\mathrm{C}-\mathrm{Cl}), 1308(\mathrm{C}-\mathrm{N}) .{ }^{1} \mathrm{H}$ NMR (400 MHz, DMSO- $\left.d_{6}, \delta, p p m\right): 6.98(\mathrm{~d}, J=16 \mathrm{~Hz}, 1 \mathrm{H}$, $\mathrm{HC}=\mathrm{CH}(\mathrm{H}-\alpha)), 7.13-7.69(\mathrm{~m}, 8 \mathrm{H}, \mathrm{Ar}-\mathrm{H}), 7.78(\mathrm{~d}, J=16 \mathrm{~Hz}, 1 \mathrm{H}$, $\mathrm{HC}=\mathrm{CH}(\mathrm{H}-\beta)), 9.60(\mathrm{~s}, 1 \mathrm{H}, \mathrm{NH})$. ESI-MS $(\mathrm{m} / \mathrm{z}): 373[\mathrm{M}+\mathrm{H}]^{+}$.

(E)-1-(4-(4,6-Dichloro-1,3,5-triazin-2-ylamino)phenyl)-3(pyridin-3-yl)-2-propen-1-one (4ee): Colour: Light yellow crystals. FT-IR $\left(\mathrm{KBr}, V_{\max } \mathrm{cm}^{-1}\right): 3122(\mathrm{~N}-\mathrm{H}), 3011(\mathrm{C}-\mathrm{H}$, aromatic), 2922 (C-H, aliphatic), 1679 (C=0), 1609 (C=C, aliphatic), 1422 (C=C, aromatic), $1308(\mathrm{C}-\mathrm{N}), 681(\mathrm{C}-\mathrm{Cl}) .{ }^{1} \mathrm{H}$ NMR (400 MHz, DMSO- $\left.d_{6}, \delta, p p m\right): 7.22(\mathrm{~d}, J=16 \mathrm{~Hz}, 1 \mathrm{H}$, $\mathrm{HC}=\mathrm{CH}(\mathrm{H}-\alpha)), 7.23-7.59(\mathrm{~m}, 8 \mathrm{H}, \mathrm{Ar}-\mathrm{H}), 7.68(\mathrm{~d}, J=16 \mathrm{~Hz}, 1 \mathrm{H}$, $\mathrm{HC}=\mathrm{CH}(\mathrm{H}-\beta)), 9.58(\mathrm{~s}, 1 \mathrm{H}, \mathrm{NH})$. ESI-MS $(\mathrm{m} / \mathrm{z}): 373[\mathrm{M}+\mathrm{H}]^{+}$.

(E)-1-(4-(4,6-Dichloro-1,3,5-triazin-2-ylamino)phenyl)-3(pyridin-4-yl)-2-propen-1-one (4ff): Colour: Light yellow crystals. FT-IR $\left(\mathrm{KBr}, v_{\max }, \mathrm{cm}^{-1}\right): 3127(\mathrm{~N}-\mathrm{H}), 3019(\mathrm{C}-\mathrm{H}$, aromatic), 2931 (C-H, aliphatic), $1689(\mathrm{C}=0), 1604$ (C=C, aliphatic), 1417 (C=C, aromatic), $688(\mathrm{C}-\mathrm{Cl}), 1308(\mathrm{C}-\mathrm{N}) .{ }^{1} \mathrm{H}$ NMR $\left(400 \mathrm{MHz}\right.$, DMSO- $\left.d_{6}, \delta, \mathrm{ppm}\right): 6.98(\mathrm{~d}, J=16 \mathrm{~Hz}, 1 \mathrm{H}$, $\mathrm{HC}=\mathrm{CH}(\mathrm{H}-\alpha)), 7.13-7.69(\mathrm{~m}, 8 \mathrm{H}, \mathrm{Ar}-\mathrm{H}), 7.78(\mathrm{~d}, J=16 \mathrm{~Hz}, 1 \mathrm{H}$, $\mathrm{HC}=\mathrm{CH}(\mathrm{H}-\beta)), 9.60(\mathrm{~s}, 1 \mathrm{H}, \mathrm{NH})$. ESI-MS $(\mathrm{m} / \mathrm{z}): 373[\mathrm{M}+\mathrm{H}]^{+}$.

(E)-1-(4-(4,6-Dichloro-1,3,5-triazin-2-ylamino)phenyl)-3(naphthalen-2-yl)-2-propen-1-one (4gg): Colour: Light yellow crystals. FT-IR $\left(\mathrm{KBr}, V_{\max }, \mathrm{cm}^{-1}\right): 3102(\mathrm{~N}-\mathrm{H}), 3015(\mathrm{C}-\mathrm{H}$, aromatic), 2926 (C-H, aliphatic), $1684(\mathrm{C}=0), 1602$ (C=C, aliphatic), 1416 (C=C, aromatic), $682(\mathrm{C}-\mathrm{Cl}) .{ }^{1} \mathrm{H}$ NMR $(400 \mathrm{MHz}$, DMSO- $\left.d_{6}, \delta, \mathrm{ppm}\right): 7.62-7.83(\mathrm{~m}, 11 \mathrm{H}, \mathrm{Ar}-\mathrm{H}), 7.87(\mathrm{~d}, J=15.2$ $\mathrm{Hz}, 1 \mathrm{H}, \mathrm{HC}=\mathrm{CH}(\mathrm{H}-\alpha)), 8.16(\mathrm{~d}, J=15.2 \mathrm{~Hz}, 1 \mathrm{H}, \mathrm{HC}=\mathrm{CH}(\mathrm{H}-\beta)$ ), $9.70(\mathrm{~s}, 1 \mathrm{H}, \mathrm{NH})$. ESI-MS $(\mathrm{m} / \mathrm{z}): 422[\mathrm{M}+\mathrm{H}]^{+}$.

(E)-1-(4-(4,6-Dichloro-1,3,5-triazin-2-ylamino)phenyl)-3(naphthalen-3-yl)-2-propen-1-one (4hh): Colour: Light yellow crystals. FT-IR $\left(\mathrm{KBr}, v_{\max }, \mathrm{cm}^{-1}\right): 3115(\mathrm{~N}-\mathrm{H}), 3019(\mathrm{C}-\mathrm{H}$, aromatic), 2931 (C-H, aliphatic), $1689(\mathrm{C}=0), 1604(\mathrm{C}=\mathrm{C}$, aliphatic), 1417 (C=C, aromatic), $688(\mathrm{C}-\mathrm{Cl}) .{ }^{1} \mathrm{H}$ NMR $(400 \mathrm{MHz}$, DMSO- $\left.d_{6}, \delta, p p m\right): 7.62-8.33(\mathrm{~m}, 11 \mathrm{H}, \mathrm{Ar}-\mathrm{H}), 7.89(\mathrm{~d}, J=15.2$ $\mathrm{Hz}, 1 \mathrm{H}, \mathrm{HC}=\mathrm{CH}(\mathrm{H}-\alpha)$ ), $8.26(\mathrm{~d}, J=15.2 \mathrm{~Hz}, 1 \mathrm{H}, \mathrm{HC}=\mathrm{CH}(\mathrm{H}-\beta)$ ), $9.71(\mathrm{~s}, 1 \mathrm{H}, \mathrm{NH})$. ESI-MS $(\mathrm{m} / \mathrm{z}): 422[\mathrm{M}+\mathrm{H}]^{+}$.

(E)-1-(4-(4,6-Dichloro-1,3,5-triazin-2-ylamino)phenyl)-3(anthracen-9-yl)-2-propen-1-one (4ii): Colour: Light yellow crystals. FT-IR $\left(\mathrm{KBr}, v_{\max }, \mathrm{cm}^{-1}\right): 3127(\mathrm{~N}-\mathrm{H}), 3019(\mathrm{C}-\mathrm{H}$, aromatic), 2931 (C-H, aliphatic), 1689 (C=0), 1604 (C=C, aliphatic), 1417 (C=C, aromatic), $688(\mathrm{C}-\mathrm{Cl}) .{ }^{1} \mathrm{H}$ NMR $(400 \mathrm{MHz}$, DMSO- $\left.d_{6}, \delta, p p m\right): 6.98-7.41(\mathrm{~m}, 13 \mathrm{H}, \mathrm{Ar}-\mathrm{H}), 7.59(\mathrm{~d}, J=15.6$ $\mathrm{Hz}, 1 \mathrm{H}, \mathrm{HC}=\mathrm{CH}(\mathrm{H}-\alpha)$ ), 8.06 (d, $J=15.6 \mathrm{~Hz}, 1 \mathrm{H}, \mathrm{HC}=\mathrm{CH}(\mathrm{H}-\beta)$ ), $9.75(\mathrm{~s}, 1 \mathrm{H}, \mathrm{NH})$. ESI-MS $(\mathrm{m} / \mathrm{z}): 472[\mathrm{M}+\mathrm{H}]^{+}$.

\subsection{Mycobacterium tuberculosis H37Rv inhibitory activity}

The Mycobacterium tuberculosis inhibitory activity of 1,3,5triazine-chalcone hybrid molecules $4 \mathbf{4 a - i i}$ were assessed against Mtb H37Rv strain using micro plate Alamar Blue assay (MABA) [81]. This methodology is non-toxic, uses a thermally stable reagent and shows good correlation with proportional and BACTEC radiometric method. Briefly, $200 \mu \mathrm{L}$ of sterile deionzed water was added to all outer perimeter wells of sterile 96 wells plate to minimized evaporation of medium in the test wells during incubation. The 96 wells plate received $100 \mu \mathrm{L}$ of the Middle brook 7H9 broth and serial dilution of compounds was made directly on plate. The final drug concentrations tested were 100 to $0.2 \mu \mathrm{g} / \mathrm{mL}$. Plates were covered and sealed with parafilm and incubated at $37^{\circ} \mathrm{C}$ for five days. After this time, 25 $\mu \mathrm{L}$ of freshly prepared 1:1 mixture of Alamar Blue reagent and $10 \%$ tween 80 was added to the plate and incubated for $24 \mathrm{~h}$. A blue color in the well was interpreted as no bacterial growth, and pink color was scored as growth. The MIC was defined as lowest drug concentration, which prevented the color change from blue to pink. The results of Mtb H37Rv inhibitory activity studies are given in Table 1.

\section{Results and discussion}

\subsection{Synthesis}

The IR spectrum of all the compounds 4a-ii exhibited the characteristic absorptions at various frequencies corresponddingly at 3310-3110 and $1640-1715 \mathrm{~cm}^{-1}$ suggesting the presence of a secondary amine group and $\alpha, \beta$-unsaturated carbonyl group respectively. In the ${ }^{1} \mathrm{H}$ NMR spectra of 1-(4(4,6-dichloro-1,3,5-triazin-2-ylamino) phenyl)-3-(substituted)2-propen-1-ones (4a-ii), a singlet integrating for one proton characteristic of the secondary amine $\mathrm{NH}$ group was observed in between $\delta$ 9.2-9.4 ppm as a broad signal. As seen in case of compound $\mathbf{4 a}$, the IR spectrum of $\mathbf{4 a}$ exhibited characteristic $-\mathrm{C}=\mathrm{C}-$ (aliphatic) and $-\mathrm{C}=\mathrm{C}-$ (aromatic) stretching bands at frequencies 1645 and $1513 \mathrm{~cm}^{-1}$, respectively. The other IR absorptions at various frequencies correspondingly at 3155 and $1688 \mathrm{~cm}^{-1}$ suggesting the presence of a secondary amino group and $\alpha, \beta$-unsaturatedketone group, respectively. The 400 $\mathrm{MHz}{ }^{1} \mathrm{H}$ NMR spectrum of the compound 4a in DMSO- $d_{6}$ as solvent with TMS as an internal standard exhibited characteristic peaks of $\mathrm{H}_{\alpha}$ and $\mathrm{H}_{\beta}$ protons of $\alpha, \beta$-unsaturated ketone bridge appeared as two doublets, one doublet at $\delta 7.78 \mathrm{ppm}$ $\left(\mathrm{H}_{\alpha}, J=15.2 \mathrm{~Hz}\right)$ and the other one at $\delta 8.01 \mathrm{ppm}\left(\mathrm{H}_{\beta}, J=15.2\right.$ $\mathrm{Hz}$ ). The large $J$ value $15.2 \mathrm{~Hz}$ of both the protons clearly reveals the trans geometry at the double bond. The distinguishhing peak of $\mathrm{NH}$ proton appears as one singlet $\delta 9.74 \mathrm{ppm}$. The ESI mass spectrum (positive ion mode) of 4 a revealed a $(\mathrm{M}+\mathrm{H})^{+}$ ion at $m / z$ 372. Based on the above spectral information the structure of the compound $\mathbf{4 a}$ was confirmed as $(Z)-1-(4-(4,6-$ dichloro-1,3,5-triazin-2-ylamino)phenyl)-3-(phenyl)-2-propen1-one [82-84].

\subsection{Mycobacterium tuberculosis H37RV inhibitory activity}

The results of in vitro Mycobacterium tuberculosis (H37Rv) inhibitory activity of the synthesized 1,3,5-triazine-chalcone hybrid molecules (4a-ii) is illustrated in (Table 1). The antitubercular activity screening data revealed that the compound $\mathbf{4 z}$ demonstrated comparatively the most potent inhibitory activity, with MIC value $3.125 \mu \mathrm{g} / \mathrm{mL}$. It is interesting to note that the compounds $\mathbf{4 e ,} \mathbf{4 p}$ and $\mathbf{4 b b}$ also showed appreciable inhibitory activity with MIC value $6.25 \mu \mathrm{g} / \mathrm{mL}$. Compound $\mathbf{4 s}$ was also showed satisfactory inhibitory activity with MIC value $12.5 \mu \mathrm{g} / \mathrm{mL}$. The other compounds such as $\mathbf{4 b}$, 4f, 4l, 4q, 4u, 4y, 4aa, 4cc and 4dd showed moderate level of activity with MIC $25 \mu \mathrm{g} / \mathrm{mL}$. 
The compounds 4a, 4d, 4g, 4h, 4j, 4k, 4m, 4r, 4v, 4w, 4x and $\mathbf{4 f f}$ exhibited comparatively reasonable inhibitory activity with MIC value $50 \mu \mathrm{g} / \mathrm{mL}$. Correspondingly, the compounds $4 \mathbf{c}$, 4i, 4n, 4o, 4t, 4ee, 4gg, 4hh and 4ii exhibited comparatively poor inhibitory activity with MIC value $100 \mu \mathrm{g} / \mathrm{mL}$ in comparison with the standard drugs (Ethambutol, MIC: 3.125 $\mu \mathrm{g} / \mathrm{mL}$; Pyrazinamide, MIC: $3.125 \mu \mathrm{g} / \mathrm{mL}$ and Streptomycin, MIC: $6.25 \mu \mathrm{g} / \mathrm{mL}$ ).

A direct revision into the Structure-Activity Relationship (SAR) of these compounds clearly exhibited the intrinsic property of Mycobacterium tuberculosis (H37Rv) inhibitory activity associated with the basic skeleton consisting of 1,3,5triazine and $\alpha, \beta$-unsaturatedketone moieties [85] with MIC values range $100-3.125 \mu \mathrm{g} / \mathrm{mL}$. It is noteworthy that the observed inhibitory activity of 1,3,5-triazine-chalcone hybrid molecules 4a-ii against Mycobacterium tuberculosis (H37Rv) revealed the importance of the type of substituted aromatic/ heteroaromatic aldehyde from which the corresponding 1,3,5triazine-chalcone hybrid molecules $\mathbf{4 a - i i}$ were obtained, which in some cases was enhanced by the influence of some substituents and decreased by some other substituents. The aromatic/heteroaromatic aldehydes derived chalcone derivatives of 1,3,5-triazine, as seen in the case of compounds followed its activity order as 4 bb (Thiophen-3-yl, MIC: 6.25 $\mu \mathrm{g} / \mathrm{mL}$ ) > 4aa (Furan-2yl, MIC: $25 \mu \mathrm{g} / \mathrm{mL}$ ), 4cc (Pyrrol-2yl, MIC: $25 \mu \mathrm{g} / \mathrm{mL}$ ), 4dd (Pyridin-2-yl, MIC: $25 \mu \mathrm{g} / \mathrm{mL}$ ) > 4a (Phenyl, MIC: $50 \mu \mathrm{g} / \mathrm{mL}$ ), 4ff (Pyridin-4-yl, MIC: $50 \mu \mathrm{g} / \mathrm{mL}$ ) > 4ee (Pyridin-3-yl, MIC: $100 \mu \mathrm{g} / \mathrm{mL}$ ), 4gg (Naphthalen-2-yl, MIC: $100 \mu \mathrm{g} / \mathrm{mL}$ ), 4hh (Naphthalen-3-yl, MIC: $100 \mu \mathrm{g} / \mathrm{mL}$ ), 4ii (Anthracen-9-yl, MIC: $100 \mu \mathrm{g} / \mathrm{mL}$ ), respectively. The compounds $4 \mathbf{z}\left(2,4-\mathrm{diFC}_{6} \mathrm{H}_{3}\right.$, MIC: $\left.3.125 \mu \mathrm{g} / \mathrm{mL}\right)>\mathbf{4 s}\left(2-\mathrm{ClC}_{6} \mathrm{H}_{4}\right.$, MIC: $12.5 \mu \mathrm{g} / \mathrm{mL})>\mathbf{4 u}\left(4-\mathrm{ClC}_{6} \mathrm{H}_{4}\right.$, MIC: $\left.25 \mu \mathrm{g} / \mathrm{mL}\right), \mathbf{4 y}\left(4-\mathrm{FC}_{6} \mathrm{H}_{4}\right.$, MIC: $25 \mu \mathrm{g} / \mathrm{mL})>\mathbf{4 v}\left(2,4-\mathrm{diClC}_{6} \mathrm{H}_{3}\right.$, MIC: $\left.50 \mu \mathrm{g} / \mathrm{mL}\right), \mathbf{4 w}(2-$ $\mathrm{FC}_{6} \mathrm{H}_{4}$, MIC: $\left.50 \mu \mathrm{g} / \mathrm{mL}\right), \mathbf{4 x}\left(3-\mathrm{FC}_{6} \mathrm{H}_{4}, \mathrm{MIC}: 50 \mu \mathrm{g} / \mathrm{mL}\right)>4 \mathrm{t}(3-$ $\mathrm{ClC}_{6} \mathrm{H}_{4}$, MIC: $100 \mu \mathrm{g} / \mathrm{mL}$ ) displayed better inhibitory potency indicating the significance of halogen substituents on the phenyl ring of 1,3,5-traizine-chalcone motif. It is also reported that the compounds substituted with electron releasing or activating groups was found to be biologically relevant and the activity order was $4 \mathbf{e}\left(2-\mathrm{OMeC}_{6} \mathrm{H}_{4}\right.$, MIC: $\left.6.25 \mu \mathrm{g} / \mathrm{mL}\right)>\mathbf{4 b}(2-$ $\mathrm{MeC}_{6} \mathrm{H}_{4}$, MIC: $\left.25 \mu \mathrm{g} / \mathrm{mL}\right)$, 4f (3-OMeC $6 \mathrm{H}_{4}$, MIC: $\left.25 \mu \mathrm{g} / \mathrm{mL}\right)$, $4 \mathbf{l}$ (2-Me,5- $\left.\mathrm{OHC}_{6} \mathrm{H}_{3}, \mathrm{MIC}: 25 \mu \mathrm{g} / \mathrm{mL}\right)>4 d\left(4-\mathrm{MeC}_{6} \mathrm{H}_{4}, \mathrm{MIC}: 50\right.$ $\mu \mathrm{g} / \mathrm{mL}), \mathbf{4 g}\left(4-\mathrm{OMeC}_{6} \mathrm{H}_{4}\right.$, MIC: $\left.50 \mu \mathrm{g} / \mathrm{mL}\right)$, 4h $\left(3-\mathrm{OHC}_{6} \mathrm{H}_{4}\right.$, MIC: $50 \mu \mathrm{g} / \mathrm{mL})$, 4j $\left(3,5-\mathrm{diOHC}_{6} \mathrm{H}_{3}\right.$, MIC: $\left.50 \mu \mathrm{g} / \mathrm{mL}\right)$, 4k (4,5$\operatorname{diOHC}_{6} \mathrm{H}_{3}$, MIC: $\left.50 \mu \mathrm{g} / \mathrm{mL}\right), \mathbf{4 m}\left(2-\mathrm{NH}_{2} \mathrm{C}_{6} \mathrm{H}_{4}\right.$, MIC: $\left.50 \mu \mathrm{g} / \mathrm{mL}\right)>$ 4c $\left(3-\mathrm{MeC}_{6} \mathrm{H}_{4}, \mathrm{MIC}: 100 \mu \mathrm{g} / \mathrm{mL}\right)$, 4i $\left(4-\mathrm{OHC}_{6} \mathrm{H}_{4}\right.$, MIC: 100 $\mu \mathrm{g} / \mathrm{mL}), 4 n\left(3-\mathrm{NH}_{2} \mathrm{C}_{6} \mathrm{H}_{4}, \mathrm{MIC}: 100 \mu \mathrm{g} / \mathrm{mL}\right)$, 4o $\left(4-\mathrm{NH}_{2} \mathrm{C}_{6} \mathrm{H}_{4}, \mathrm{MIC}\right.$ : $100 \mu \mathrm{g} / \mathrm{mL}$ ), respectively. The compounds substituted with electron withdrawing or deactivating nitro group was found to be biologically relevant and the activity order was, $\mathbf{4 p}$ (2$\mathrm{NO}_{2} \mathrm{C}_{6} \mathrm{H}_{4}$, MIC: $\left.6.25 \mu \mathrm{g} / \mathrm{mL}\right)>4 \mathbf{q}\left(3-\mathrm{NO}_{2} \mathrm{C}_{6} \mathrm{H}_{4}\right.$, MIC: $\left.25 \mu \mathrm{g} / \mathrm{mL}\right)>$ $4 \mathbf{r}\left(4-\mathrm{NO}_{2} \mathrm{C}_{6} \mathrm{H}_{4}, \mathrm{MIC}: 50 \mu \mathrm{g} / \mathrm{mL}\right)$, respectively. It is reported that considerable activity was observed when the hydroxyl groups are substituted at different positions on the phenyl ring as seen in the case of compounds $4 \mathbf{l}$ (2-Me, $\left.5-\mathrm{OHC}_{6} \mathrm{H}_{3}, \mathrm{MIC}: 25 \mu \mathrm{g} / \mathrm{mL}\right)>$ 4h $\left(3-\mathrm{OHC}_{6} \mathrm{H}_{4}, \mathrm{MIC}: 50 \mu \mathrm{g} / \mathrm{mL}\right), \mathbf{4 j}\left(3,5-\mathrm{diOHC}_{6} \mathrm{H}_{3}, \mathrm{MIC}: 50\right.$ $\mu \mathrm{g} / \mathrm{mL}), 4 \mathbf{k}\left(4,5-\mathrm{diOHC}_{6} \mathrm{H}_{3}, \mathrm{MIC}: 50 \mu \mathrm{g} / \mathrm{mL}\right)>\mathbf{4 i}\left(4-\mathrm{OHC}_{6} \mathrm{H}_{4}\right.$, MIC: $100 \mu \mathrm{g} / \mathrm{mL}$ ), respectively.

\section{Conclusion}

A series of new class of Mycobacterium tuberculosis H37Rv inhibitors is reported, the synthesis of which is characterized by conventional methods. During this study we have identified a number of 1,3,5-triazine-chalcone hybrid molecules (4a-ii) empowered with significant Mycobacterium tuberculosis H37Rv inhibitory properties. Structure activity relationship studies revealed that substitution at position 3 of $\alpha, \beta$-unsaturated ketone further substitution is important to modulate the activity. Further studies determining the in vivo antitubercular activity of these compounds are under progress.

\section{Acknowledgements}

One of the authors Mr. Dwarampudi Subbarayal Reddy is thankful to the Principal, Andhra University College of Pharmaceutical Sciences, Andhra University, Visakhapatnam, for providing necessary facilities in carrying out the research work.

\section{References}

[1]. World Health Organization, www.who.int/tb.

[2]. Janin, Y. L. Bioorg. Med. Chem. 2007, 15, 2479-2513.

[3]. Gutierrez-Lugo, M. T.; Bewley, C. J. Med. Chem. 2008, 51, 2606-2612.

[4]. Manetti, F.; Magnani, M.; Castagnolo, D.; Passalacqua, L.; Botta, M.; Corelli, F.; Saddi, M.; Deidda, D.; De Logu, A. Chem. Med. Chem. 2006, 1, 973-989.

[5]. Barnes, P. F.; Blotch, A. B.; Davidson, P. T.; Snider, Jr. D. E.; N. Engl. J. Med. 1991, 324, 1644-1649.

[6]. Sbarbaro, J. A. Chest. 1997, 111, 1149-1150

[7]. Fujiwara, P. I.; Cook, S. V.; Rutherford, C. M.; Crawford, J. T.; Glickman S. E.; Kreiswirth, B. N.; Sachdev, O. S. S.; Ebrahimzadeh, A.; Frieden, T. R. Arch. Intern. Med. 1997, 157, 531-535.

[8]. Bibikova, M. V.; Borisova, N. A.; Orekhov, S. N.; Katlinskiŭ, A. V. Antibiot Khimioter. 2006, 51(1), 22-27.

[9]. Bernstein, J.; Lott, W. A.; Steinberg, B. A.; Yale, H. L. Am. Rev. Tuberc. 1952, 65, 357-364.

[10]. Patel, R. V.; Kumari, P.; Rajani, D. P.; Chikhalia, K. H. Eur. J. Med. Chem. 2011, 46, 4354-4365.

[11]. Pastorin, G.; Federico, S.; S. Paoletta, S.; Corradino, M.; Cateni, F.; Cacciari, B.; Klotz, K.; Gao, Z.; Jacobson, K. A.; Spalluto, G.; Moro, S. Bioorg. Med. Chem. 2010, 18, 2524-2536.

[12]. Pastorin, G.; Federico, S.; Paoletta, S.; Corradino, M.; Cateni, F.; Cacciari, B.; Klotz, K.; Gao, Z.; Jacobson, K. A.; Spalluto, G.; Moro, S. Bioorg. Med. Chem. 2010, 18, 2524-2536.

[13]. Popowycz, F.; Schneider, C.; DeBonis, S.; Skoufias, D. A.; Kozielski, F.; Galmarini, C. M.; Joseph, B. Bioorg. Med. Chem. 2009, 17, 3471-3478.

[14]. Sunduru, N.; Agarwal, A.; Katiyar, S. B.; Nishi, N.; Goyal, N.; Guptab, S.; Chauhana, P. M. S. Bioorg. Med. Chem. 2006, 14, 7706-7715.

[15]. Vilaivan, T.; Saesaengseerung, N.; Jarprung, D.; Kamchonwongpaisan, S.; Sirawaraporn, W.; Yuthavong, Y. Bioorg. Med. Chem. 2003, 11, $217-$ 224.

[16]. Zhou, C.; Min, J.; Liu, Z.; Young, A.; Deshazer, H.; Gao, T.; Chang, Y. T.; Kallenbach, N. R. Bioorg. Med. Chem. Lett. 2008, 18, 1308-1311.

[17]. Krecmerova, M.; Masojidkova, M.; Holy, A. Bioorg. Med. Chem. 2010, 18, 387-395.

[18]. Sunduru, N.; Gupta, L.; Chaturvedi, V.; Dwivedi, R.; Sinha, S.; Chauhan, P. M. S. Eur. J. Med. Chem. 2010, 45, 3335-3345.

[19]. Garaj, V.; Puccetti, L.; Fasolis, G.; Winum, J.; Montero, J.; Scozzafava, A.; Vullo, D.; Innocentia, A.; Supurana, C. T. Bioorg. Med. Chem. Lett. 2005, 15, 3102-3108.

[20]. Sosic, I.; Mirkovi, B.; Turk, S.; Stefane, B.; Kos, J.; Gobec, S. Eur. J. Med. Chem. 2011, 46, 4648-4656.

[21]. Xia, Y.; Mirzai, B.; Chackalamannil, S.; Czamiecki, M.; Wang, S.; Clemmons, A.; Ahn, H.; Boykow, G. C. Bioorg. Med. Chem. Lett. 1996, 6 919-922.

[22]. Fitzgerald, L.; Miller, K.; Marshallyy, W. J. Bioorg. Med. Chem. 2003, 11, 4093-4102.

[23]. Zuev, D.; Mattson, R. J.; Huang, H.; Mattson, G. K.; Zueva, L.; Nielsen, J. M.; Kozlowski, E. S.; Huang, X. S.; Wua, D.; Gao, Q.; Lodge, N. J.; Bronson, J. J.; Macor, J. E. Bioorg. Med. Chem. Lett. 2011, 21, 24842488.

[24]. Gopalsamy, A.; Yang, H.; Ellingboe, J. W.; McKew, J. C.; Tam, S.; McCarthy, D.; Zhang, W.; Shen, M.; Clarke, J. D. Bioorg. Med. Chem. Lett. 2006, 16, 2978-2981.

[25]. Andrews, K. M.; Beebe, D. A.; Benbow, J. W.; Boyer, D. A.; Doran, S. D.; Hui, Y.; Liu, S.; Kirk McPherson, R.; Neagu, C.; Parker, J. C.; Piotrowski, D. W.; Schneider, S. R.; Treadway, J. L.; VanVolkenberg, M. A.; Zembrowski, W. J. Bioorg. Med. Chem. Lett. 2011, 21, 1810-1814.

[26]. McKay, G. A.; Reddy, R.; Arhin, F. S; Belley, A.; Lehoux, D.; Moeck, G.; Sarmiento, I.; Parr, T. R.; Gros, P.; Pelletier, J.; Fara, A. R. Bioorg. Med. Chem. Lett. 2006, 16, 1286-1290.

[27]. Dehnhardt, C. M.; Venkatesan, A. M.; Chen, Z.; Santos, D. E.; Kaloustian A. S.; Brooijmans, N.; Yu, K.; Hollander, I.; Feldberg, L.; Lucas, J.; Mallon, R. Bioorg. Med. Chem. Lett. 2011, 21, 4773-4778.

[28]. Huang, W.; Zheng, W.; Urban, D. J.; Inglese, J.; Sidransky, E.; Austina, C P.; Thomasa, C. J. Bioorg. Med. Chem. Lett. 2007, 17, 5783-5789.

[29]. Park, H.; Hwang, K. Y.; Kim, Y. H.; Oh, K. H.; Lee, J. Y.; Kim, K. Bioorg. Med. Chem. Lett. 2008, 18, 3711-3715.

[30]. Courme, C.; Gresh, N.; Vidal, M.; Lenoir, C.; Garbay, C.; Florent, J.; Bertounesque, E. Eur. J. Med. Chem. 2010, 45, 244-255.

[31]. Guo, Z.; Wu, D.; Zhu, Y. F.; Tucci, F. C.; Pontillo, J.; Saunders, J.; Xie, Q. Struthers, R. S.; Chena, C. Bioorg. Med. Chem. Lett. 2005, 15, 693-702.

[32]. Mattson, R. J.; Denhart, D. J.; Catt, J. D.; Dee, M. F.; Deskus, J. A.; Ditta, J. L.; Epperson, J.; King, H. D.; Gao, A.; Poss, M. A.; Purandare, A.; 
Tortolani, D.; Zhao, Y.; Yang, H.; Yeola, S.; Palmer, J.; Torrente, J.; Stark, A.; Johnson, G. Bioorg. Med. Chem. Lett. 2004, 14, 4245-4248.

[33]. Pitts, W. J. Guo, J; Dhar, T. G. M. Shen, Z; Gu, H. H. Watterson, S. H. Bednarz, M. S.; Chen, B.; Barrish, J. C.; Bassolino, D.; Cheney, D.; Fleener, C. A.; Rouleau, K. A.; Hollenbaugh, D. L.; Iwanowicz, E. J. Bioorg. Med. Chem. Lett. 2002, 12, 2137-2142.

[34]. Richard, D. J.; Verheijen, J. C.; Yu, K.; Zask, A. Bioorg. Med. Chem. Lett. 2010, 20, 2654-2657.

[35]. Bregman, H.; Nguyen, H. N.; Feric, E.; Ligutti, J.; Liu, D.; McDermott, J. S.; Wilenkin, B.; Zou, A.; Huang, L.; Li, X.; McDonough, S. I.; DiMauro, E. F. Bioorg. Med. Chem. Lett. 2012, 22, 2033-2042.

[36]. Maa, X.; Poon, T. Y.; Hon Wong, P. T.; Chui, W. K. Bioorg. Med. Chem. Lett. 2009, 19, 5644-5647.

[37]. Raboisson, P.; Schultz, D.; Muller, C.; Reimund, J.; Pinna, G.; Mathieu, R.; Bernard, P.; Do, Q.; DesJarlais, R. L.; Justiano, H.; Lugnier, C.; Bourguignon, J. Eur. J. Med. Chem. 2008, 43, 816-829.

[38]. Nie, Z.; Perretta, C.; Erickson, P.; Margosiak, S.; Lu, J.; Averill, A.; Almassy, R.; Chua, S. Bioorg. Med. Chem. Lett. 2008, 18, 619-623.

[39]. Ho, K. K.; Beasley, J. R.; Belanger, L.; Black, D.; Chan, J.; Dunn, D.; Hu, B.; Klon, A.; Kultgen, S. G.; Ohlmeyer, M.; Parlato, S. M.; Ray, P. C.; Pham, Q.; Rong, Y.; Roughton, A. L.; Walker, T. L.; Wright, J.; Xu, K.; Xu, Y.; Zhang, L.; Webba, M. Bioorg. Med. Chem. Lett. 2009, 19, 6031-6035.

[40]. Xu, W.; Chen, G.; Liew, O. W.; Zuo, Z.; Jiang, H.; Zhu, W. Bioorg. Med. Chem. Lett. 2009, 19, 3188-3192.

[41]. Mylari, B. L.; Withbroe, G. J.; Beebe, D. A.; Brackett, N. S.; Conn, E. L.; Coutcher, J. B.; Oates, P. J.; Zembrowski, W. J. Bioorg. Med. Chem. 2003, 11, 4179-4188.

[42]. Jin, H.; Cianchetta, G.; Devasagayaraj, A.; Gua, K.; Marinelli, B.; Samala, L.; Scott, S.; Stouch, T.; Tunoori, A.; Wang, Y.; Zang, Y.; Zhang, C.; Kimball, S. D.; Main, A. J.; Ding, Z.; Sun, W.; Yang, Q.; Yu, X.; Powell, D. R.; Wilson, A.; Liu, Q.; Shi, Z. Bioorg. Med. Chem. Lett. 2009, 19, 52295232.

[43]. Porter, J. R. Archibald, S. C. Brown, J. A.; Childs, K.; Critchley, D.; Head, J. C.; Hutchinson, B.; Parton, T. A. H.; Robinson, M. K.; Shock, A.; Warrellow, G. J.; Zomaya, A. Bioorg. Med. Chem. Lett. 2002, 12, 15911594.

[44]. Jarrahpour, A.; Khalili, D.; De Clercq, E.; Salmi, C.; Brunel, J. M. Molecules 2007, 12, 1720-1730.

[45]. Perumal, P.; Bilal, A. R.; Dontireddy, R. S. R.; Natesh, R. K. Eur. J. Med. Chem. 2009, 44, 2328-2340.

[46]. Kui, C.; Qing, Z.; Yong, Q.; Lei, S.; Jing, Z.; Hai, Z. Bioorg. Med. Chem. 2009, 17, 7861-7871.

[47]. Panchal, P. K.; Pansuriya, P. B.; Patel, M. N. J. Enzyme Inhib. Med. Chem. 2006, 21, 453-458.

[48]. Jarrahpour, A.; Khalili, D.; De, C. E.; Salmi, C.; Brunel, J. M. Molecules 2007, 12, 1720-1730.

[49]. Koneni, V. S.; Jammikuntla, N. R.; Gitika, B.; Saxena, J. K. Eur. J. Med. Chem. 2008, 43, 2592-2596.

[50]. Vanco, J.; Svajlenova, O.; Racanska, E.; Muselik, J.; Valentova, J. J. Trace Elem. Med. Biol. 2004, 18, 155-161.

[51]. Ambike, V.; Adsule, S.; Ahmed, F.; Wang, Z.; Afrasiabi, Z.; Sinn, E.; Sarkar, F.; Padhye, S. J. Inorg. Biochem. 2007, 101, 1517-1524.

[52]. Sinha, D.; Tiwari, A. K.; Singh, S.; Shukla, G.; Mishra, P.; Chandra, H.; Mishra, A. K. Eur. J. Med. Chem. 2008, 43, 160-165.

[53]. Gihane, N.; Eddy, P.; Claudiu, T. S.; Jean-Yves, W.; Mihail, B. Bioorg. Med. Chem. Lett. 2009, 19, 6014-6017.

[54]. You, Z.; Shi, D.; Xu, C.; Zhang, Q.; Zhu, H. Eur. J. Med. Chem. 2008, 43, 862-871.

[55]. Amin, R.; Krammer, B.; Abdel-Kader, N.; Verwanger, T.; El-Ansary, A. Eur. J. Med. Chem. 2010, 45, 372-378.

[56]. Pradeepkumar, M. R.; Malleshappa, N. N.; Sheetal, S.; Satyanarayana, D.; Veeresh, S. M. Eur. J. Med. Chem. 2010, 45, 85-89.

[57]. Zahid, H. C.; Hassan, M.; Khalid, M. K.; Claudiu, T. S. J. Enzyme Inhib. Med. Chem. 2005, 20, 183-188.

[58]. Bharti, S. K.; Nath, G.; Tilak, R.; Singh, S. K. Eur. J. Med. Chem. 2010, 45, 651-660

[59]. Ye, X.; Chen, Z.; Zhang, A.; Zhang, L. Molecules. 2007, 12, 1202-1209.

[60]. Gacche, R. N.; Gond, D. S.; Dhole, N. A.; Dawane, B. S. J. Enzyme Inhib. Med. Chem. 2006, 21, 157-161.

[61]. Toyato, E.; Sekizaki, H.; Itoh, K.; Tanizawa, K. Chem. Pharm. Bull. 2003, 51, 625-629.

[62]. Cardile, V.; Panico, A. M.; Geronikaki, A.; Gentile, B.; Ronsisvalle, G. Farmaco 2002, 57, 1009-1013.

[63]. Doddareddy, M. R.; Cho, Y. S.; Koh, H. Y.; Pae, A. N. Bioorg. Med. Chem. 2004, 12, 3977-3985.

[64]. Jayashree, B. S.; Anuradha, D.; Venugopala, K. N. Asian J. Chem. 2005, 17, 2093-2097.

[65]. Bhandari, S. V.; Bothara, K. G.; Raut, M. K.; Patil, A. A.; Sarkate, A. P.; Mokale, V. J. Bioorg. Med. Chem. 2008, 16, 1822-1831.

[66]. Venugopala, K. N.; Jayashree, B. S. Asian J. Chem. 2004, 16, 407-411.

[67]. Sondhi, S. M.; Singh, N.; Kumar, A.; Meijer, O. L. L. Bioorg. Med. Chem. 2006, 14, 3758-3765.

[68]. Dinkova, K. A. T.; Massiah, M. A.; Bozak, R. E.; Hicks, R. J.; Talalay P. P. Natl. Acad. Sci. USA 2001, 98, 3404-3409.

[69]. Nakagawa, G.; Lee, K. Tetrahedron Lett. 2006, 47, 8263-8265.
[70]. Narender, T.; Shweta, S.; Gupta S. Bioorg. Med. Chem. Lett. 2004, 14(15), 3913-3916.

[71]. Nielsen, S. F.; Chen, M.; Theander, T. G.; Kharazmi, A.; Christensen, S. B. Bioorg. Med. Chem. Lett. 1995, 5, 449-452.

[72]. Wu, J. H.; Wang, X. H.; Lee, K. H. Bioorgan. Med. Chem. 2003, 13, 1813-1815.

[73]. Wu, X.; Wilairat, P.; Go, M. Bioorg. Med. Chem. Lett. 2002, 12, 2299-2302.

[74]. Phrutivorapongkul, A.; Kirtikaro, K. Chem. Pharm. Bull. 2003, 51(6), 746-748.

[75]. Kumar, S. K.; Hager, E.; Pettit, C.; Gurulingappa, H.; Davidson, N. E.; Khan, S. R. J. Med. Chem. 2003, 46, 2813-2815.

[76]. Narender, T.; Khaliq, T.; Shweta, N.; Gupta, S. Bioorgan. Med. Chem. 2005, 13, 6543-6550

[77]. Nakaido, T.; Ohmoto, T. Chem. Pharm. Bull. 1984, 32(12), 4929-4934.

[78]. Kostanekki, S. T.; Szabranki, W. Ber. Dtsch. Chem. Ges. 1904, 37 2634-2635.

[79]. Jun, N.; Hong, G.; Jun, K. Bioorgan. Med. Chem. 2007, 15(6), 2396-2402.

[80]. Vasudeva, R. A.; Rajendra, P. Y.; Venkateswara, R. P.; Kishore, N. K. Venkata, M. R. P.; Prasad, C.; Venkateswara, R. G.; Bhavani, B. Bioorg. Med. Chem. 2013, 23, 5968-5970.

[81]. Maria, C. S. L.; Marcus, V. N.; Alessandra, C. P.; Marcelle, L. F.; Goncalves, T, M.; Nogneira, M. A. P. Arkivoc 2007, 15, 181-191.

[82]. Somayajulu, N.; Divakara, L.; Kasapu, V. V. V. S.; Rao, A.; Bugata, B.; Yenupuri, S. Eur. J. Chem. 2014, 5(1), 144-149.

[83]. Bugata, B.; Krishna, K.; Satya, V.; Avupati, V. R.; Gavalapu, V.; Somayajulu, N.; Divakara, L.; Barla, S. Eur. J. Chem. 2013, 4(4), 396 401.

[84]. Raval, J. P. Eur J. Chem. 2011, 2(3), 388-393.

[85]. Hu, Z.; Ting, M.; Zhe, C.; Ziqi, Y.; Guolin, Z.; Yijia, L.; Yongping, Y. J. Combin. Chem. 2009, 11(2), 267-273. 